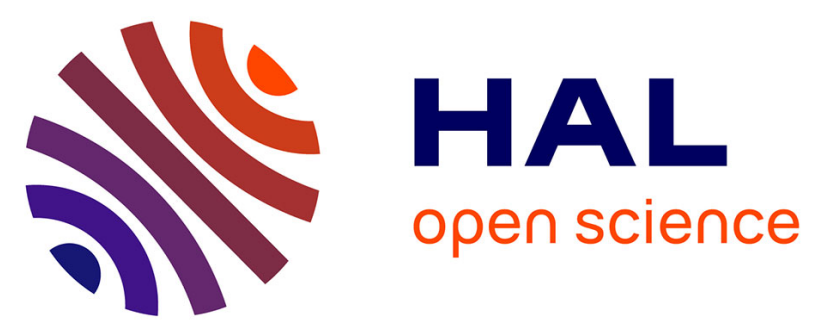

\title{
The FEMapproach using a mixed hexahedral finite element to model the electromagnetic and mechanical behavior of radiative microstrip antennas
}

\author{
Nicolas Adnet, Isabelle Bruant, F. Pablo, Laurent Proslier
}

\section{- To cite this version:}

Nicolas Adnet, Isabelle Bruant, F. Pablo, Laurent Proslier. The FEMapproach using a mixed hexahedral finite element to model the electromagnetic and mechanical behavior of radiative microstrip antennas. Engineering Analysis with Boundary Elements, 2014, 38, pp.17-30. 10.1016/j.enganabound.2013.09.012 . hal-01421509

\section{HAL Id: hal-01421509 \\ https://hal.parisnanterre.fr/hal-01421509}

Submitted on 14 Jan 2019

HAL is a multi-disciplinary open access archive for the deposit and dissemination of scientific research documents, whether they are published or not. The documents may come from teaching and research institutions in France or abroad, or from public or private research centers.
L'archive ouverte pluridisciplinaire HAL, est destinée au dépôt et à la diffusion de documents scientifiques de niveau recherche, publiés ou non, émanant des établissements d'enseignement et de recherche français ou étrangers, des laboratoires publics ou privés. 


\title{
The FEM-BIM approach using a mixed hexahedral finite element to model the electromagnetic and mechanical behavior of radiative microstrip antennas
}

\author{
N. Adnet, I. Bruant*, F. Pablo, L. Proslier \\ Laboratoire d'Energétique, Mécanique et Electromagnétisme, Université Paris Ouest Nanterre La Défense, EA 4416, \\ 50 rue de Sèvres, 92410 Ville d'Avray, France
}

Keywords:

Microstrip antenna

Electromagnetic/mechanical coupling

FEM/BIM approach

Radiation pattern

Input impedance

Reflexion coefficient

\begin{abstract}
A B S T R A C T
This work is focused on the prediction of the impact of microstrip radiative antenna distortions on electromagnetic fields. In this way, a recent numerical tool, able to model the electromagnetic and mechanical behavior of a microstrip antenna, has been developed. Considering a weak coupling between electromagnetism and mechanical behavior, the mechanical equations are first solved. Then, from the mechanical strains results for the antenna, the electromagnetic fields are computed. To solve both problems, a 3D hexahedral finite element is used to discretize the structure, having both nodal mechanical and edges electromagnetic degrees of freedom. The weak electromagnetic formulation inducing integrals on an open infinite domain, a Boundary Integral Method (BIM) is used and applied to the strained structure. Three examples are considered. The simulations show that mechanical distortions can have major influence on the electromagnetic behavior of antennas.
\end{abstract}

\section{Introduction}

Many studies focused on embedding antennas on aircraft structural surfaces have been performed over the last 15 years in order to improve both structural efficiency and antenna performances. Nowadays, microstrip antennas which are low-profile and conformable to planar and warped surfaces can easily be bounded on aircraft structural surfaces. They seem to be an interesting solution. Moreover, the emergence of metamaterials (new electromagnetic materials) will soon allow researchers to elaborate multifrequency microstrip antennas [1,2].

In flight conditions, planes are subjected to aerodynamic loads leading to the structure distortion. Hence, the influences of these structural surface distortions on the microstrip electromagnetic response have to be studied, independent of antenna developments.

A few papers dealing with curved antennas can be found in the literature. In Antilla and Alexopoulos [3], a 3D hybrid finiteelement-integral equation method has been developed in general curvilinear coordinates. In Jacobs [4], planar and curved radiative microstrip antennas are, for example, analysed. In Khedrouche et al. [5], a multilayer cylindrical-rectangular microstrip radiative patch antenna is considered. The influence of the curvature on the radar cross section is moreover shown for receiving antenna in

\footnotetext{
* Corresponding author. Tel.: +33140974856.

E-mail address: isabelle.bruant@u-paris10.fr (I. Bruant).
}

Volakis et al. [6]. Nevertheless, only simple configurations like cylindrical surfaces are considered in these works. In operating conditions, the distorted surfaces of the antenna being complex, specific tools have to be developed. On the other hand, an overview of industrial coupling softwares (like ANSYS, COMSOL) has shown that these tools are not well suited to solve this kind of coupled problem $[7,8]$. The novelty of this work is to mix the mechanical and electromagnetic problems, using the same finite element, in order to take into account the physical distortion of the radiative antenna.

The present work, focused on the development of a numerical tool able to predict the impact of microstrip distortions on electromagnetic fields, is part of the MSIE project (Intelligent Materials and Structures for Electromagnetism), launched in 2008 by the competitive French cluster AStech with the view of answering to the above problem.

The study of electromagnetic/mechanical coupling modeling for scattering microstrip antennas has been developed in [9]. Here, it is presented for the analysis of radiative microstrip antennas. It derives from the usual mechanical approach and specific mathematic tools for free space problems $[6,10]$. From a 3D discretization of the structure, a classical mechanical finite nodal element model is coupled with an electromagnetic finite vector element one, developed by Jin [11]. This leads to a particular finite element having both nodal mechanical and edges electromagnetic degrees of freedom. Hence, the electromagnetic/mechanical problem is solved using the same mesh. In order to solve mechanical problems with warped or distorted antennas, the considered finite element is an hexahedral 
one. The electromagnetic problem is solved using the boundary Integral Method (BIM) applied to the strained structure.

In Section 2, the microstrip antenna integrated on a composite structure is described and the solving strategy is introduced. In Section 3 , the solution of the mechanical problem is succinctly presented. Section 4 deals with the solution of the electromagnetic equations, in particular with the electromagnetic field approximation, using the finite vector element approach and the boundary integral method. In the last section, this numerical tool is used for several configurations.

\section{Problem description}

\subsection{Geometric description}

The specific structure to be considered in the present work is illustrated in Fig. 1. It consists of a rectangular microstrip patch antenna printed on a dielectric substrate. A ground plane is glued underneath the substrate in order to stop electromagnetic waves propagation. The antenna thickness being smaller than the substrate one will be neglected in the following developments. This structure is then supposed to be integrated on composite aircraft panels.

The cavity model described in Jin [11] and illustrated in Fig. 2 can be used to represent the above structure. The ground plane is then considered as an infinite metallic plane and the substrate is within the entire cavity. Studying radiative antennas, the patch element is excited using a coaxial probe feed.

In the following developments the infinity space located above the cavity and the ground plane, and inside of the cavity will respectively be represented by $V_{\infty}$ and $V$ symbols.

Considering the Cartesian coordinate system $(O, \hat{x}, \hat{y}, \hat{z})$ presented in Fig. 3:

- any point $M^{\prime}\left(x^{\prime}, y^{\prime}, z^{\prime}\right)$ of the infinity space is located by the vector $\overrightarrow{r^{\prime}}=\overrightarrow{O M^{\prime}}$ whose Euclidian norm is defined by $r^{\prime}=$

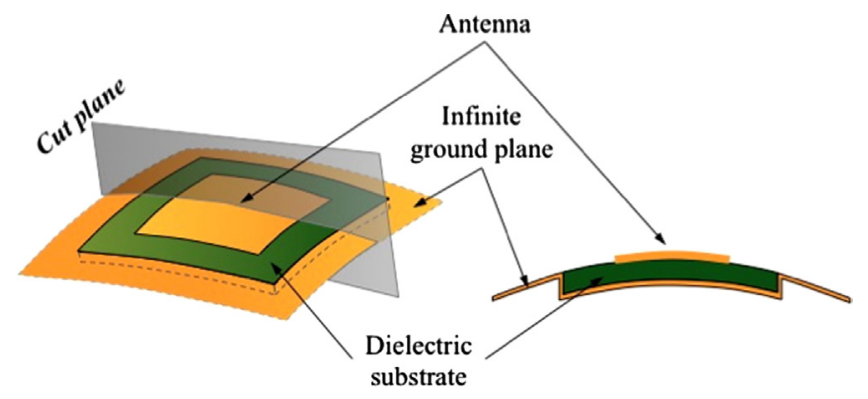

Fig. 1. The studied structure.

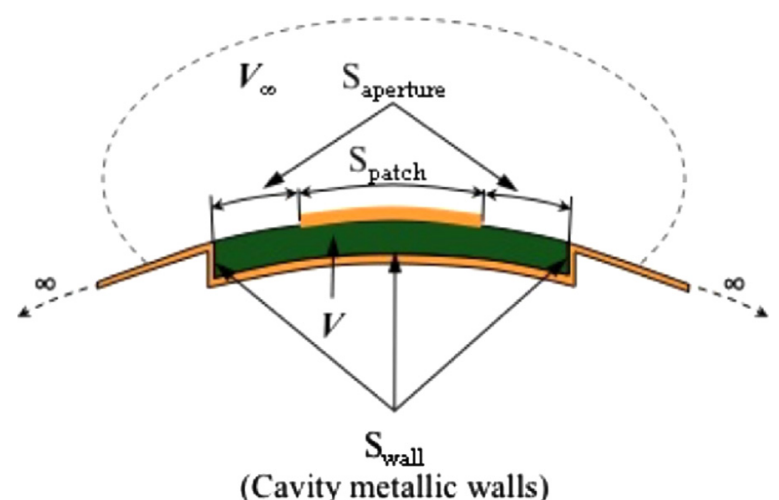

Fig. 2. The cavity model.

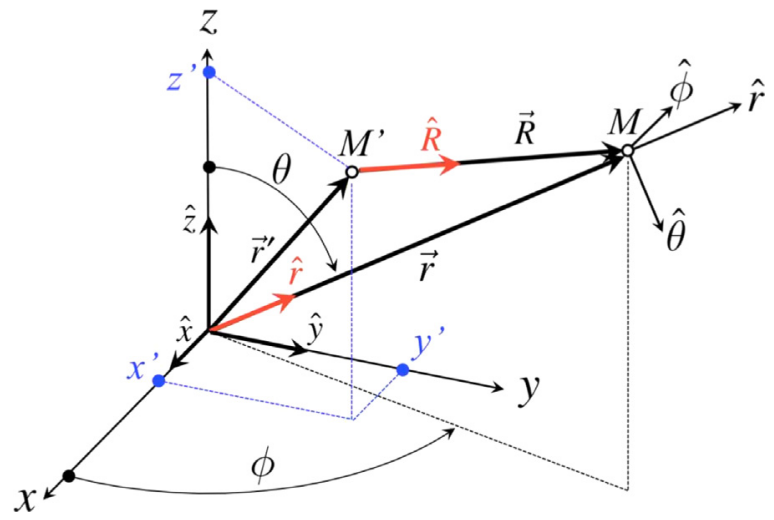

Fig. 3. The coordinate system.

$\left\|\vec{r}^{\prime}\right\|=\sqrt{x^{\prime 2}+y^{\prime 2}+z^{\prime 2}}$ (note that $M^{\prime}$ is located by $M^{\prime}\left(r^{\prime}, \theta^{\prime}, \phi^{\prime}\right)$ in the spherical frame $(\hat{\rho}, \hat{\theta}, \hat{\phi}))$;

- the $\vec{E}^{\text {int }}$ and $\vec{H}^{\text {int }}$ fields are the internal electric and magnetic fields due to the antenna feeds respectively. They are received by the observation point $M$ of the infinity space, located by the vector $\vec{r}=\overrightarrow{O M}$.

Finally, it is assumed that the cavity is subjected to mechanical loads, inducing mechanical strains and distortions.

\subsection{Solution strategy}

As the antenna is excited by a coaxial probe feed and also subjected to mechanical loads, the studied problem is a coupled one. In the present study, the mechanical behavior is supposed to be non-sensitive to electromagnetic fields. Thus, a weak coupling between electromagnetism and mechanical behavior is assumed in this work. Then, the coupled problem is processed, at each calculation step, as follows:

- first, the mechanical problem is solved;

- then, the electromagnetic problem is solved, taking into account the mechanical strains of the structure.

The main difficulty in the analysis of an electromagnetic problem for antennas consists of estimating integrals on an open infinite domain. Using numerical methods such as the Finite Element Method (FEM), the discretized domain to be considered has to be extended far from the source region in order to impose the radiation condition. This leads to huge computing times.

Meanwhile, it is possible to avoid this problem using the Finite Element-Boundary Integral (FEM-BIM) Method [11]. This method consists of introducing a fictitious boundary which encloses the structures to be studied. Interior to this boundary, the Finite Element Method is used to formulate the fields, whereas in the exterior region, the fields are represented by a boundary integral. The fields of the two regions are coupled at the fictitious boundary via the fields continuity conditions. This leads to a coupled system for solution of the interior and boundary fields.

Finally, both mechanical and electromagnetic problems can be solved using finite element discretization. The specificity of the present method lies in the use of a single finite element having both nodal mechanical and edges electromagnetic degrees of freedom. The mechanical and electromagnetic problems are then solved using the same mesh, at each calculation step.

In order to ensure the solution of the electromagnetic problem to meet the four Maxwell's equations in the three directions, and 
for a better consideration of electromagnetic boundary condition, the microstrip antenna needs to be discretized using 3D finite elements. For meshes compatibility reasons the substrate is also discretized using 3D finite elements.

Five main kinds of elements shape can be considered: brick, tetrahedra, prism, pyramid and hexahedra. But, the hexahedral elements (or distorted brick element), able to automatically mesh arbitrary structures, are best suited to model the warped antennas. This kind of elements is not usually used in electromagnetic problems. Here, we use one of them to solve mechanical and electromagnetic equations.

In the next two sections, we briefly present the mechanical and electromagnetic solutions.

\section{Solution of the mechanical problem}

As previously underlined, the structural cavity $V$ will be bounded on composite panels which are distorted by external loads. Hence, this distortion will affect $V$. The study of its mechanical strains is then essential.

\subsection{Mechanical governing equations and weak form}

Considering a static approach, the mechanical governing equations consist of the equilibrium equation, the constitutive equation in $V$ and the strain-displacement relationship [12]:

$\overrightarrow{\operatorname{div}} \mathbf{T}+\vec{f}=\overrightarrow{0}$,

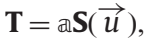

$\mathbf{S}(\vec{u})=\frac{1}{2}\left(\operatorname{grad} \vec{u}+\operatorname{grad}^{T} \vec{u}\right)$,

where $\mathbf{T}, \mathbf{S}(\vec{u}), \vec{f}$ and a are respectively the stress tensor, the strain tensor, the prescribed body forces vector applied to $V$ and the elastic stiffness tensor. In addition to these equations, one has to take into account the mechanical boundary conditions: the first one where mechanical vector displacement $\vec{u}$ is imposed, and the second one where a surface force vector $\vec{F}$ is applied on the boundary $\partial V_{F}$.

The classical weak form of the mechanical problem is then given by, find $\vec{u} \in \mathcal{U}_{\text {ad }}$ such that

$\int_{V} \mathbf{S}^{*}\left(\vec{u}^{*}\right): \mathbf{T}(\vec{u}) d V=\int_{V} \vec{u}^{*} \cdot \vec{f} d V+\int_{\partial V_{F}} \vec{u}^{*} \cdot \vec{F} d S$,

for any admissible virtual displacement $\vec{u}^{*} \in \mathcal{U}_{a d}^{*}$.

This variational principle is the usual starting point for any finite element approximations in mechanical modeling.

\subsection{The finite element approximation for the mechanical part}

The element implemented in the software developed for the present study is similar to the ANSYS SOLID45 element [13]. It consists of an 8-node linear brick element with three degrees of freedom at each node (three translations in the nodal directions).

\subsubsection{The elemental matrices}

Here classical methods are used. Using the engineering formalism, the strain and stress tensors can be written as vectors:

$\vec{S}=\{S\}=\left\{S_{x x} S_{y y} S_{z z} S_{x z} S_{y z} S_{x y}\right\}^{T}$

$\vec{T}=\{T\}=\left\{T_{x x} T_{y y} T_{z z} T_{x z} T_{y z} T_{x y}\right\}^{T}$

Then, the stiffness tensor $₫$ can be written as a $6 \times 6$ matrix $\mathbf{a}$.
Discretizing the studied structure using the Finite Element Method, nodal displacements and strains of any element are approximated by

$\{u\}=\mathbf{N}\left\{q_{e}\right\}$ and $\quad\{S\}=\mathbf{B}\left\{q_{e}\right\}$

where the vector $\left\{q_{e}\right\}$ contains the element nodal displacements and $\mathbf{N}$ and $\mathbf{B}$ are $6 \times 24$ matrices respectively containing the interpolation functions and their derivatives.

Substituting these relationships in each integral of the weak formulation (4) restrained to elementary volume $V_{e}$ and surface $S_{e}$, the following elemental stiffness matrix and loads vector are obtained:

$\mathbf{K}^{e}=\int_{V_{e}} \mathbf{B}^{T} \mathbf{a B} d V_{e}$

$\left\{F^{e}\right\}=\int_{V_{e}} \mathbf{N}^{T}\{f\} d V_{e}+\int_{S_{e}} \mathbf{N}^{T}\{F\} d S_{e}$

where $\{f\}$ and $\{F\}$ are the vectors containing the body and surface loads applied to the element nodes.

\subsubsection{The discretized problem}

Assembling the elemental matrices over the volume, the mechanical problem can be expressed in the following usual global form:

$\mathbf{K}\{q\}=\left\{F_{\text {ext }}\right\}$

where $\{q\}$ contains the mechanical displacement degrees of freedom of the structure and $\left\{F_{\text {ext }}\right\}$ corresponds to the applied external mechanical loads.

The stiffness matrix $\mathbf{K}$ being symmetric and the equation being linear, classical numerical schemes can be used to solve the mechanical problem.

\section{Solution of the electromagnetic problem}

This section is dedicated to the electromagnetic problem for a radiative antenna. From the weak formulation, the FEM-BIM technique is used to determine the electromagnetic fields in the whole space.

\subsection{Electromagnetic governing equations}

Considering a radiative antenna, the electromagnetic governing equations in the $V_{\infty}$ space and the cavity $V$ consist of the well known Maxwell's equations [11,14] and the constitutive relationships. In addition, the boundary conditions, radiation conditions, jump conditions and electromagnetic excitation are considered for the mathematical formulation.

The usual time harmonic approach is considered in the following developments. As a consequence, all field quantities are supposed to be harmonically oscillating functions with a single frequency $\omega[11,14]$.

\subsubsection{The constitutive relations}

They describe the macroscopic properties of the mediums

$\vec{D}=\varepsilon \vec{E}$

$\vec{B}=\mu \vec{H}$,

where $\vec{E}, \vec{D}, \vec{H}$ and $\vec{B}$ are respectively the electric field intensity, the electric flux density, the magnetic field intensity and the magnetic flux density. The constitutive parameters $\varepsilon$ and $\mu$ are respectively the permittivity and permeability of the medium. These parameters are scalars for isotropic media. 
In the present study, the cavity backed patch antenna is considered to be situated in free space. Then, $V_{\infty}$ is characterized by the permittivity $\varepsilon_{0}$ and the permeability $\mu_{0}$. Moreover, the cavity is supposed to be filled with an homogeneous material represented by a relative permittivity $\varepsilon_{r}=\varepsilon / \varepsilon_{0}$ and a relative permeability $\mu_{r}=\mu / \mu_{0}$. These two properties are here scalars but would have been tensors in the case of inhomogeneous filling material.

\subsubsection{The radiation conditions}

If the outer boundary of a domain recedes to infinity, this domain is called unbounded or open. In this case, to make sure that the solution of the problem is unique, an additional condition has to be specified at this outer boundary. It is referred to a radiation condition as the Sommerfeld gauge [11]:

$\lim _{r \rightarrow \infty} r\left[\overrightarrow{r o t} \vec{E}+j k_{0} \vec{r} \wedge \vec{E}\right]=\overrightarrow{0}$,

$\lim _{r \rightarrow \infty} r\left[\overrightarrow{r o t} \vec{H}+j k_{0} \vec{r} \wedge \vec{H}\right]=\overrightarrow{0}$,

where $k_{0}$ is the free-space wavenumber $\left(k_{0}=2 \pi / \lambda_{0}=\omega \sqrt{\varepsilon_{0} \mu_{0}}\right)$.

\subsubsection{The jump conditions}

In the present problem, the conservation laws imply to meet the following jump conditions across the interface between the two medias $V_{\infty}$ and $V[15]$ :

$\vec{n} \cdot[[\vec{D}]]=0$,

$\vec{n} \cdot[[\vec{B}]]=0$,

$\vec{n} \wedge[[\vec{E}]]=\overrightarrow{0}$,

$\vec{n} \wedge[[\vec{H}]]=\overrightarrow{0}$,

where $\vec{n}$ is the normal vector of the interface. For a function $g$, the symbol $[[\cdot]]$ is defined as $[[g]]=g_{V_{\infty}}-g_{V}$.

\subsubsection{Electromagnetic excitation}

Considering a radiative antenna, the electromagnetic excitation rises from internal sources within the cavity.

It consists of the term $\vec{J}_{\text {int }}(\vec{r})$ in the Maxwell equations, which denotes the internal electric source due to the antenna feed. Here, the coaxial feed is modeled as a vertical probe of constant current $I_{0}[11]$, located at $\vec{r}_{f}$.

The expression of $\vec{J}_{\text {int }}(\vec{r})$ is

$\vec{J}_{\text {int }}(\vec{r})=\delta\left(\vec{r}-\vec{r}_{f}\right) I_{0} e^{j \phi_{0}} \ln$

where $l$ is the length of the probe feed, $\hat{n}$ its unitary orientation vector (the coaxial feed is assumed to be orthogonal to $S_{\text {aperture }}$ ), and $\phi_{0}$ is the phase of the applied electric field.

In the next sections, the usual approach which consists of solving the problem according to the electric field $\vec{E}$ is followed. Once $\vec{E}$ is established, the magnetic flux density can be obtained using the first relationship of Maxwell's equations.

\subsection{Weak electromagnetic formulation}

The analytical developments of this electromagnetic problem are detailed in Jin [11]. From the Maxwell equations and Eqs. (11)-(19), the weak formulation of the electromagnetic boundary value problem is [9]: find $\vec{E} \in \mathcal{E}_{\text {ad }}$ such that

$F_{v}\left(\vec{E}, \vec{E}^{*}\right)+F_{s}\left(\vec{E}, \vec{E}^{*}\right)=F_{\text {int }}\left(\vec{E}^{*}\right)$ for all admissible electric field $\vec{E}^{*} \in \mathcal{E}_{a d}^{*}$, with

$$
\begin{aligned}
& F_{v}\left(\vec{E}, \vec{E}^{*}\right)= \int_{V} \overrightarrow{\operatorname{rot}} \vec{E}^{*}(\vec{r}) \frac{1}{\mu_{r}} \overrightarrow{r o t} \vec{E}(\vec{r}) d V \\
&-k_{0}^{2} \int_{V} \vec{E}^{*}(\vec{r}) \varepsilon_{r} \vec{E}(\vec{r}) d V \\
& F_{S}\left(\vec{E}, \vec{E}^{*}\right)=\left.2 \int_{S_{a p}} \int_{S_{a p}} \operatorname{div}\right|_{r}\left(\hat{n} \wedge \vec{E}_{s}^{*}(\vec{r})\right) G_{0}\left(\vec{r}, \vec{r}^{\prime}\right) \ldots \\
&\left.\operatorname{div}\right|_{r^{\prime}}\left(\hat{n}^{\prime} \wedge \vec{E}_{S}\left(\vec{r}^{\prime}\right)\right) d S^{\prime} d S-2 k_{0}^{2} \int_{S_{a p}} \int_{S_{a p}} G_{0}\left(\vec{r}, \vec{r}^{\prime}\right) \ldots \\
&\left(\hat{n} \wedge \vec{E}_{S}^{*}(\vec{r})\right) \cdot\left(\hat{n}^{\prime} \wedge \vec{E}_{S}\left(\vec{r}^{\prime}\right)\right) d S^{\prime} d S \\
& F_{\text {int }}\left(\vec{E}^{*}\right)=-j k_{0} Z_{0} \int_{V_{\text {feed }}} \vec{E}^{*}(\vec{r}) \vec{J}_{\text {int }}(\vec{r}) d V
\end{aligned}
$$

where $S_{a p}=S_{\text {aperture }}$ denotes the area of the cavity which is not covered by the antenna (see Fig. 2), $\hat{v}$ its unit normal vector, $\vec{E}_{s}$ the surface electric field and $Z_{0}=\sqrt{\mu_{0} / \varepsilon_{0}} . V_{\text {feed }}$ includes all coaxial feeds. The bilinear function $F_{s}$ depends on the scalar Green's function $G_{0}\left(\vec{r}, \vec{r}^{\prime}\right)$, given by [16-19]

$G_{0}\left(\vec{r}, \vec{r}^{\prime}\right)=\frac{e^{-j k_{0}\left|\vec{r}-\vec{r}^{\prime}\right|}}{4 \pi\left|\vec{r}-\vec{r}^{\prime}\right|}$

In this weak form, the integrals reduce to the inside of the cavity and the aperture surface. Nevertheless, the solution of this formulation will also give the far fields $\vec{H}^{\text {far }}$ and $\vec{E}^{\text {far }}$, using the following relation [6], $\forall|\vec{r}| \geq 1 / k_{0}$ :

$\vec{E}^{f a r}(\vec{r})=-z_{0}\left(\vec{n} \wedge \vec{H}^{f a r}(\vec{r})\right)$

$\vec{H}^{f a r}(\vec{r})=j \frac{k_{0}}{z_{0}} \int_{S_{a p}} \mathbb{G}\left(\vec{r}, \vec{r}^{\prime}\right) \cdot\left(\vec{n}^{\prime} \wedge \vec{E}_{S}\left(\vec{r}^{\prime}\right)\right) d S^{\prime}$

where $\mathbb{G}\left(\vec{r}, \vec{r}^{\prime}\right)$ is the dyadic Green's function, evaluated for the far zone, given by

$\mathbb{G}\left(\vec{r}, \vec{r}^{\prime}\right)=\left(\llbracket-\frac{1}{k_{0}^{2}} \overrightarrow{\operatorname{grad}^{\prime}} \overrightarrow{\operatorname{grad}^{\prime}}\right) G_{0}\left(\vec{r}, \vec{r}^{\prime}\right)$,

here $\mathbb{~}$ is the unity dyadic function [16-19] and the symbol ' means that the operator is attached to the variable $\vec{r}^{\prime}$.

\subsection{The finite element approximation for the electromagnetic part}

Node based expansions for finite element solutions are suitable for modeling scalar quantities but typically not so for simulating electromagnetic vector fields. As a consequence, a new approach, recently used in electromagnetism, uses the vector finite elements or edge elements which assign degrees of freedom to the edges rather than to the nodes of the elements. These types of elements have been described by Krantz [20]. They have been shown to be free of the shortcomings of node-based expansions [11,21]. Especially, the occurrence of non-physical solutions also called spurious solutions and generally attributed to lack of enforcement of the divergence condition is avoided. Several investigations and applications can be found in the literature $[6,10,21,22]$.

\subsubsection{Vector finite elements}

The electromagnetic problem represented by the weak formulation (20) will be solved using a classical hexahedral edge element presented in Fig. 4. 


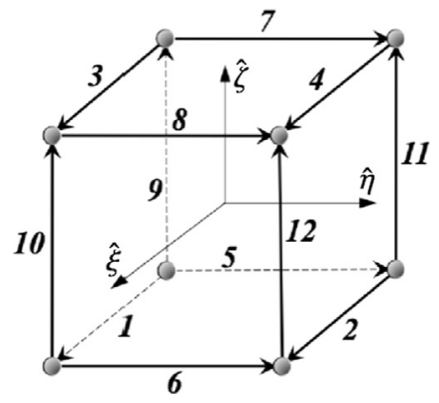

Fig. 4. The finite element.

Here, $\xi, \eta$ and $\zeta$ represent the reduced coordinates, varying in $[-1 ; 1]^{3}$, in the $(0, \hat{\xi}, \hat{\eta}, \hat{\zeta})$ system of reference. Each edge is assigned by a number $i$ :

- $i=1-4$ for edges parallel to $\hat{\xi}$,

- $i=5-8$ for edges parallel to $\hat{\eta}$,

- $i=9-12$ for edges parallel to $\hat{\zeta}$.

The electrical field in the element is then approximated by

$\vec{E}^{e}=\sum_{i=1}^{12} \overrightarrow{W_{i}^{v}}(\xi, \eta, \zeta) E_{i}^{e}$

The functions $\overrightarrow{W_{i}^{v}}$ are the basis functions and are given in Appendix A. The use of these basic functions leads to two important features to be underlined:

- the continuity of the tangential field across all element edges is guaranteed,

- the divergence condition $\operatorname{div} \overrightarrow{W_{i}^{v}}=0$ within the element is satisfied.

Eq. (28) can thus be written in a matrix form as

$\vec{E}^{e}=\left\{\begin{array}{l}E_{x}^{e} \\ E_{y}^{e} \\ E_{z}^{e}\end{array}\right\}=\mathbf{W}_{\mathbf{v}}\left\{E^{e}\right\}$

with

$\mathbf{W}_{\mathbf{v}}=\left[\begin{array}{llll}\overrightarrow{W_{1}^{v}} \cdot \hat{x} & \overrightarrow{W_{2}^{v}} \cdot \hat{x} & \ldots & \overrightarrow{W_{12}^{v}} \cdot \hat{x} \\ \overrightarrow{W_{1}^{v}} \cdot \hat{y} & \overrightarrow{W_{2}^{v}} \cdot \hat{y} & \ldots & \overrightarrow{W_{12}^{v}} \cdot \hat{y} \\ \overrightarrow{W_{1}^{v}} \cdot \hat{z} & \overrightarrow{W_{2}^{v}} \cdot \hat{z} & \ldots & \overrightarrow{W_{12}^{v}} \cdot \hat{z}\end{array}\right]$

and $\left\{E^{e}\right\}$ is the vector of components $E_{i}^{e}$ (size $\left.12 \times 1\right)$.

Considering the weak formulation equation (20), two kinds of terms can be distinguished: terms depending on volume integrals and terms depending on surface ones. All integrals are estimated using the above hexahedral edge element. Meanwhile, it is necessary to impose that one coordinate equals to one for surface integrals. The basis functions $\overrightarrow{W_{i}^{s}}$ so obtained are gathered in the $\mathbf{W}_{s}$ matrix.

\subsubsection{The elemental matrices}

Substituting Eq. (29) into each integral of Eq. (20) restrained to elementary volume $V_{e}$ and surface $S_{e}$ gives the following elemental matrices for a curved structure:

$\mathbf{K}_{\mathbf{e}}^{\mathbf{v}}=\int_{V_{e}} \mathbf{W}_{\mathbf{v}}^{\mathbf{T}} \mathbf{R O T}^{\mathbf{T}} \boldsymbol{\mu}_{\mathbf{r}}^{-1} \mathbf{R O T} \mathbf{W}_{\mathbf{v}}^{\mathbf{T}} d V_{e}$

$\mathbf{M}_{\mathbf{e}}^{\mathbf{v}}=\int_{V_{e}} \mathbf{W}_{v}^{T} \varepsilon_{\mathbf{r}} \mathbf{W}_{v} d V_{e}$
$\mathbf{M}_{\mathbf{e}}^{\mathbf{s}}=2 \int_{S_{e}} \int_{S_{e}} \mathbf{W}_{s}^{T} \mathbf{N}^{\mathbf{T}} G_{0}\left(\vec{r}, \vec{r}^{\prime}\right) \mathbf{N}^{\prime} \mathbf{W}_{s} d S_{e}^{\prime} d S_{e}$

$\mathbf{K}_{\mathbf{e}}^{\mathbf{s}}=2 \int_{S_{e}} \int_{S_{e}} \mathbf{W}_{s}^{T} \mathbf{N}^{\mathbf{T}} \mathbf{D I V}^{\mathbf{T}} G_{0}\left(\vec{r}, \vec{r}^{\prime}\right) \mathbf{D I V} \mathbf{N}^{\prime} \mathbf{W}_{s} d S_{e}^{\prime} d S_{e}$

$\left\{F_{e}^{e x t}\right\}=Z_{0} l I_{0} e^{j \phi_{0}} \mathbf{W}_{v}^{T}\left(\vec{r}_{f}\right) \hat{n}$

where ROT is the rotational operator:

$\mathbf{R O T}=\left[\begin{array}{ccc}0 & -\partial / \partial z & \partial / \partial y \\ \partial / \partial z & 0 & -\partial / \partial x \\ -\partial / \partial y & \partial / \partial x & 0\end{array}\right]$

$\mathbf{N}$ is the matrix associated with $\hat{n} \wedge$, with $\hat{n}=n_{x} \hat{x}+n_{y} \hat{y}+n_{z} \hat{z}$

$\mathbf{N}=\left[\begin{array}{ccc}0 & -n_{y} & n_{z} \\ n_{z} & 0 & -n_{x} \\ -n_{y} & n_{x} & 0\end{array}\right]$

and DIV is the divergence operator, defined by

$\mathbf{D I V}=\left[\begin{array}{lll}\partial / \partial x & \partial / \partial y & \partial / \partial z\end{array}\right]$

$\mathbf{N}^{\prime}$ and DIV' are respectively associated with the source point $M^{\prime}$. Their expressions are similar to $\mathbf{N}$ and DIV.

\subsubsection{The discretized problem}

Assembling the elemental matrices over the microstrip antenna volume, the electromagnetic problem can be expressed in a very global compact form as follows:

$\mathbf{Y}\left\{q_{E}\right\}=\{F\}_{e m a g}$

where $\left\{q_{E}\right\}$ contains the electric field degrees of freedom, and

$\mathbf{Y}=k_{0}^{2}\left(\mathbf{M}^{\mathbf{v}}+\mathbf{M}^{\mathbf{s}}\right)+\left(\mathbf{K}^{\mathbf{v}}+\mathbf{K}^{\mathbf{s}}\right)$

$\{F\}_{\text {emag }}=j k_{0}\left\{F^{\text {ext }}\right\}$

$\mathbf{M}^{\mathbf{v}}, \mathbf{M}^{\mathbf{s}}, \mathbf{K}^{\mathbf{v}}, \mathbf{K}^{\mathbf{s}}$ and $\{F\}_{\text {emag }}$ are the generalized matrix and vector. This relationship is similar to the classical static mechanical one. In the electromagnetic case the equivalent stiffness matrix is made up of two terms:

- $k_{0}^{2} \mathbf{M}^{\mathbf{v}}+\mathbf{K}^{\mathbf{v}}$ which is the contribution of the antenna structure discretized using the Finite Element Method,

- $k_{0}^{2} \mathbf{M}^{\mathbf{s}}+\mathbf{K}^{\mathbf{s}}$ which is the contribution of the space, discretized using the Boundary Integral Method.

Matrices $\mathbf{M}^{\mathbf{v}}, \mathbf{K}^{\mathbf{v}}, \mathbf{M}^{\mathbf{s}}$ and $\mathbf{K}^{\mathbf{s}}$ being symmetric and Eq. (39) being linear, its resolution seems to be easy. Meanwhile the evaluation of these last two matrices needs some attention: the green function, which has to be integrated, presents singularities at some particular points of the elements. This difficulty can be avoided using different Gauss points when integrating with respect to surfaces $S$ and $S^{\prime}$.

Moreover, $k_{0}^{2} \mathbf{M}^{\mathbf{s}}+\mathbf{K}^{\mathbf{s}}$ is a full matrix. In order to reduce computing times, it is converted into a sparse matrix using usual numerical technics [6].

\section{Numerical applications}

Here, the use of the FEM/BIM approach to model distorted radiative antenna is considered.

In the next applications, several electromagnetic parameters are determined from the electromagnetic fields, to evaluate the effect of mechanical strains on the electromagnetic behavior:

- the input impedance: $Z_{i j}=R_{i j}+j X_{i j}$,

- the reflexion coefficient: $S_{i j}$ 
where $i$ and $j$ respectively denote the considered patch $i$ and connector $j$. These parameters characterize the ability of the antenna to receive (through the probes) and radiate a maximum power, for its defined frequency.

At this frequency, the amplitude of $S_{i i}$ should be as lower as possible and $Z_{i i}$ should be real and maximum (to a wanted value). The analysis of $Z_{i j}$ and $S_{i j}$ allows to study the interactions between several radiative patches of an antenna. Of course, the objective is to minimize them.

In all simulations, the electromagnetic excitation is characterized by a unit current $\left(I_{0}=1\right)$ and a null phase $\left(\phi_{0}=0\right)$. Moreover, the mechanical prescribed body force is also considered equal to zero.

\subsection{Validation test: a planar antenna}

In this first test, a planar antenna, studied in [11,25], is considered to validate our model. It consists of a one patch antenna, presented in Figs. 5 and 6. It is fed by a coaxial probe located at $x=12.2 \mathrm{~mm}$ and $y=8.5 \mathrm{~mm}$, and a $50 \Omega$ charge is applied to the patch at $x=-22 \mathrm{~mm}$ and $y=-15 \mathrm{~mm}$. The substrate dielectric permittivity equals to 2.17 and a loss tangent is considered, equals to 0.0015 .

Figs. 7 and 8 show the input impedance for the antenna. The finite element results are compared with measured data for a coaxially fed microstrip antenna, given in [25]. The numerical tool developed in this work gives a good approximation of

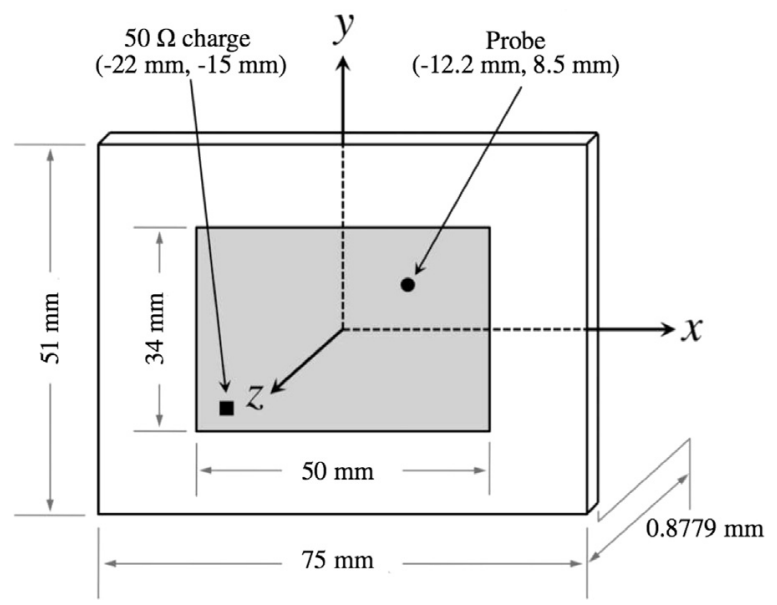

Fig. 5. A planar one patch antenna.

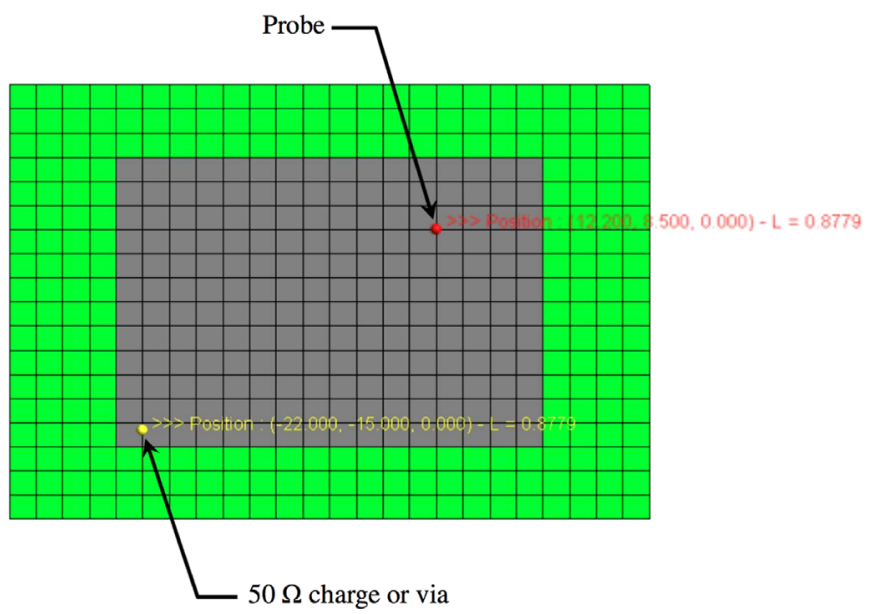

Fig. 6. The mesh of the one patch antenna.

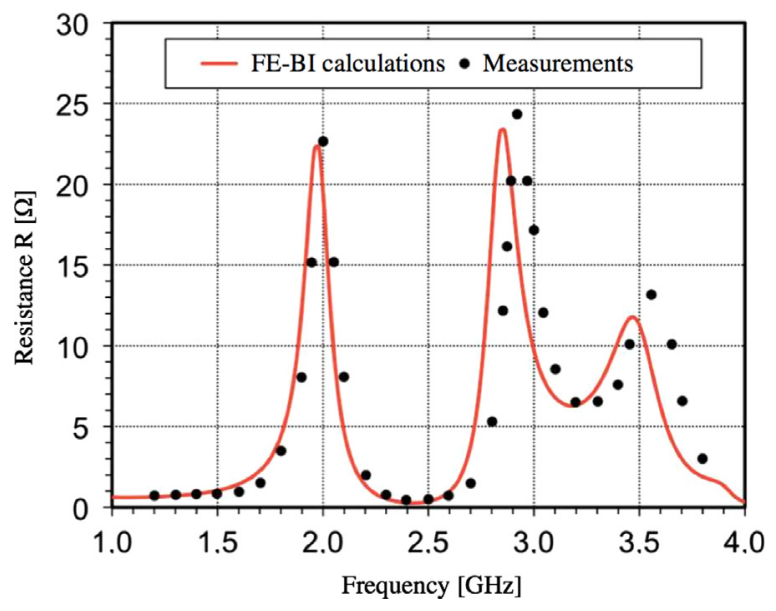

Fig. 7. Input impedance - resistance part.

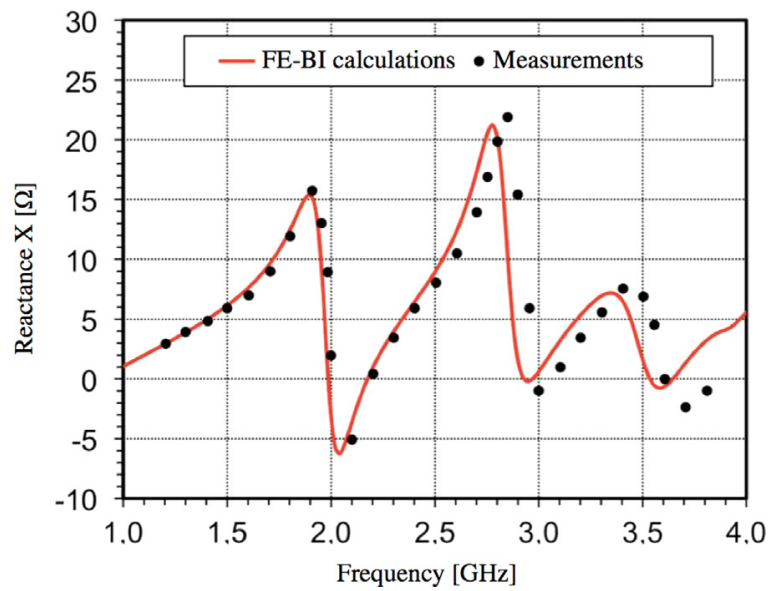

Fig. 8. Input impedance - reactance part.

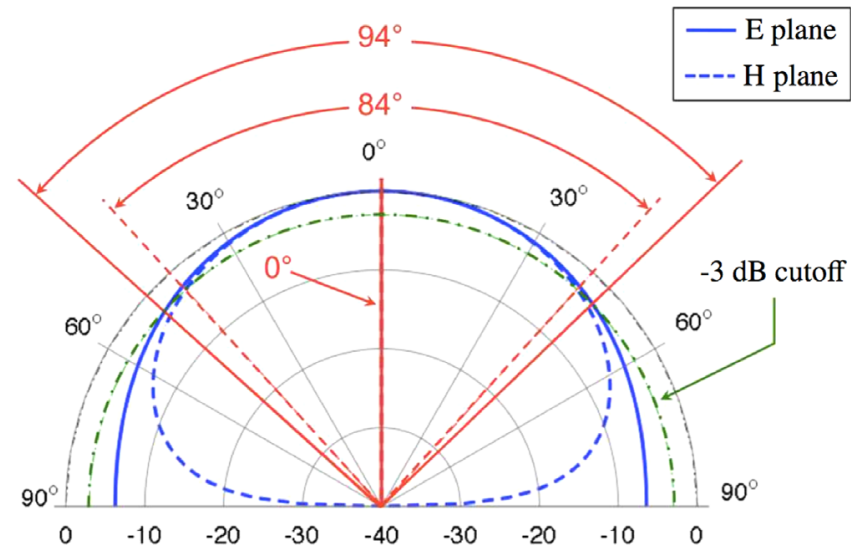

Fig. 9. Radiation patterns with hexahedral finite element.

measurements. Similar results are presented in [11], where brick elements are used.

The radiation pattern of the unloaded patch at its first resonant frequency is shown in Figs. 9 and 10 respectively with our hexahedral finite element and the brick element developed in [11]. Curves are also similar.

Other validation tests of the electromagnetic edge finite element are detailed in $[9,26]$. The next applications deal with deformed structures. 


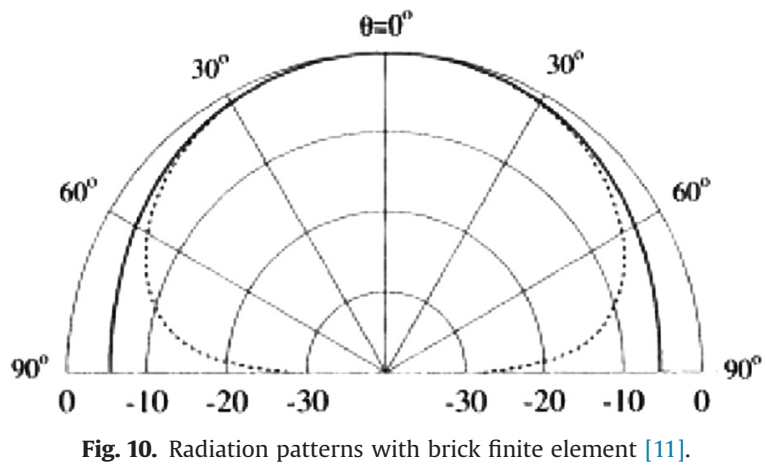

Fig. 10. Radiation patterns with brick finite element [11]

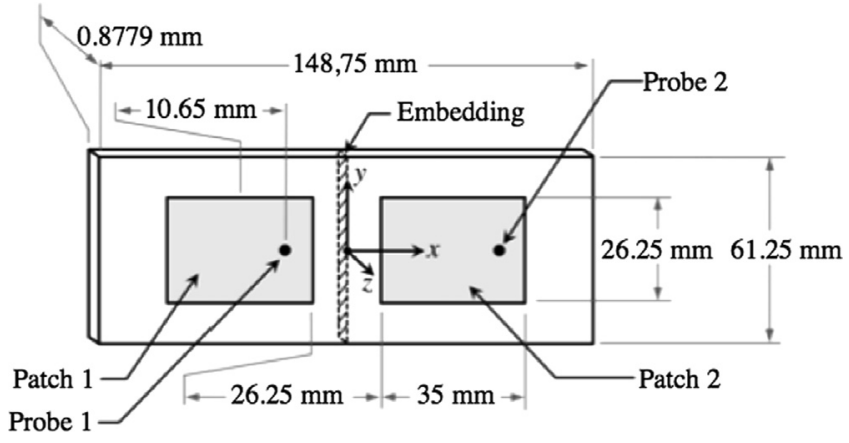

Fig. 11. A 2 patches antenna.

\subsection{A 2 patches antenna}

Here, the radiative antenna presented in Fig. 11 is considered. It consists of two patches printed on a dielectric substrate, whose permittivity and Young modulus are respectively equal to 2.32 and $1 \mathrm{GPa}$, fed by probes located on their right hand side. The antenna is embedded through the thickness on its middle line $(x=0)$ and adapted to $260 \Omega$.

Three antenna shapes are hereafter considered:

- case 1: planar antenna,

- case 2: conformed antenna, obtained by conforming the substrate on a $R=60 \mathrm{~cm}$ radius cylindrical surface before printing,

- case 3: strained antenna, obtained by applying a sinusoidal pressure such that its resulting curvature is similar to the conformed antenna one. Here, the applied pressure equals to

$$
P=P_{0}\left|\sin \left(\frac{\pi x}{L}\right)\right| \text {, }
$$

where $L$ is the length of the dielectric substrate and $P_{0}$ equals to $0.007 \mathrm{MPa}$.

Whatever the considered antenna shape, calculations were performed using $34 \times 14$ edge meshes as presented in Figs. 12-14.

The input impedances and reflexion coefficients of each port have been calculated on the $[2.45-2.63 \mathrm{GHz}]$ frequency range using a $2 \mathrm{MHz}$ step size. Results are plotted in Figs. 15 and 16 for the planar, conformal and strained configurations.

First, whatever the shape configuration considered, impedance and reflexion coefficients are merged for the two ports: $Z_{11}=Z_{22}$ and $S_{11}=S_{22}$. The geometrical symmetry of the antennas with respect to the middle line implies these curves to be similar.

On the other hand, the conformation and mechanical distortion effects on the previous coefficients are opposite compared to the planar results. Indeed, conformation leads to a shift in curves

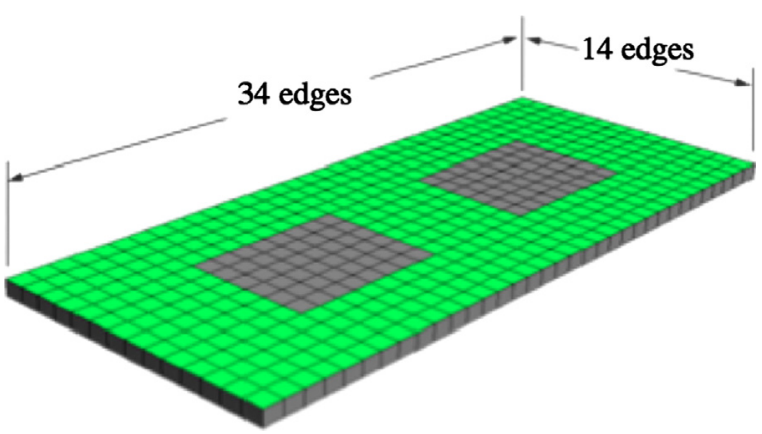

Fig. 12. Meshed planar antenna.

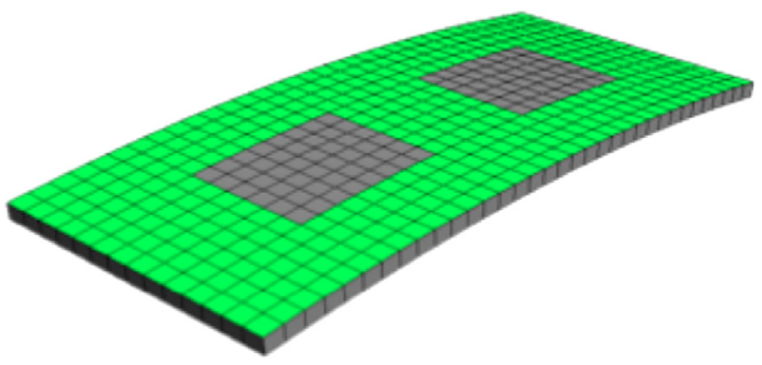

Fig. 13. Meshed conformed antenna.

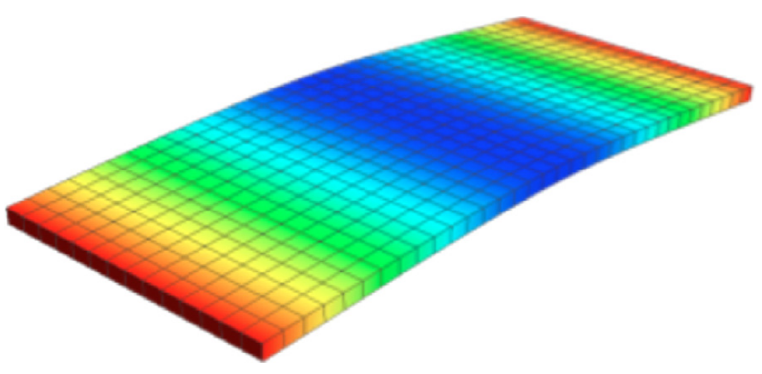

Fig. 14. Mechanical displacement of the strained antenna.

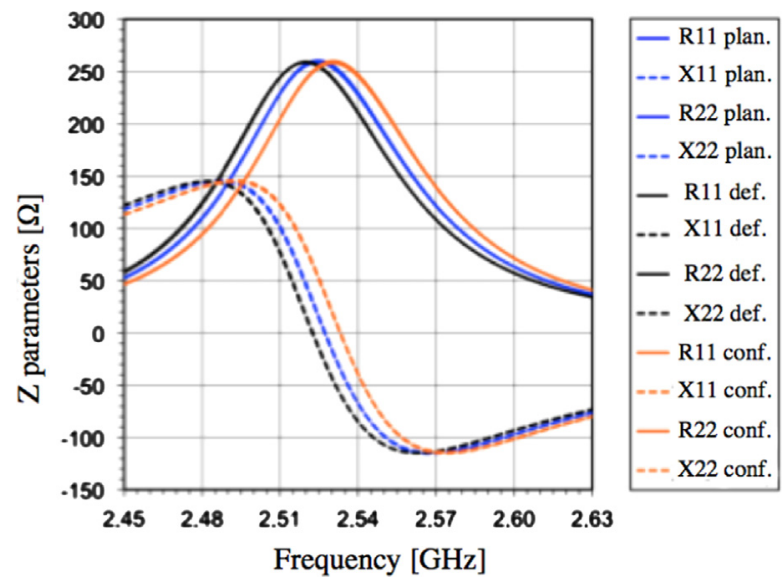

Fig. 15. Input impedances.

toward the right while distortion induces a shift toward the left. These phenomena could be explained as follows:

- in case 3, the mechanical load leads to patch extensions. As the resonant frequency of a patch antenna is inversely proportional to its width $[27,28]$, this frequency shifts to lower values, 


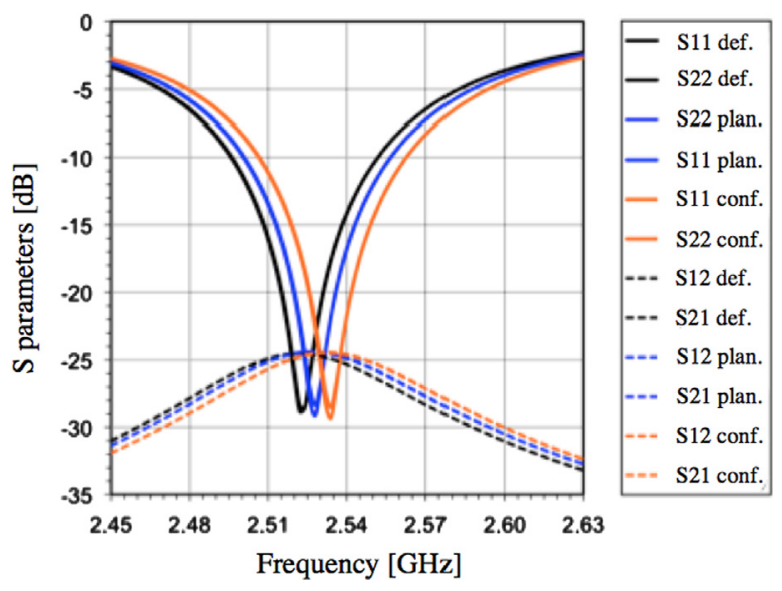

Fig. 16. Reflexion coefficients.

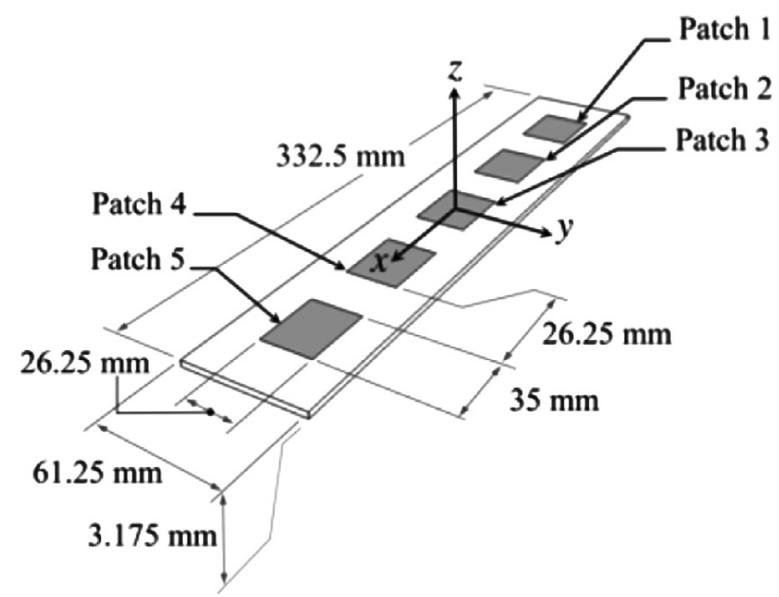

Fig. 17. Cantilever 5 patches antenna.

- in case 2, patches are printed after substrate conformation. Their dimensions are then similar to planar antenna ones. On the other hand, the substrate curvature may lead to a decrease of its apparent dielectric permittivity. The resonant frequency being inversely proportional to this parameter [27,28] may induce a shift toward higher values. Such aspects have been pointed out in [29].

\subsection{A radiative 5 patches antenna}

In this application, an antenna studied in [23,24] for planar and conformal configurations, is considered. It consists of five $35 \mathrm{~mm} \times 26.15 \mathrm{~mm}$ patches, regularly spaced, lying on the top of a $332.5 \mathrm{~mm}(L) \times 61.25 \mathrm{~mm} \times 3.175 \mathrm{~mm}$ dielectric substrate, as presented in Fig. 17. The substrate dielectric permittivity and Young modulus respectively equal to 2.32 and $1 \mathrm{GPa}$. Finally, each patch is fed by a coaxial probe located at $10.65 \mathrm{~mm}$ in the $x$-direction from its center.

\subsubsection{Cantilever antenna}

The antenna is here embedded on $x=-L / 2$ and is subjected to two local loads $F=0.05 \mathrm{~N}$, applied at the two corners on $x=L / 2$ as presented in Fig. 18.

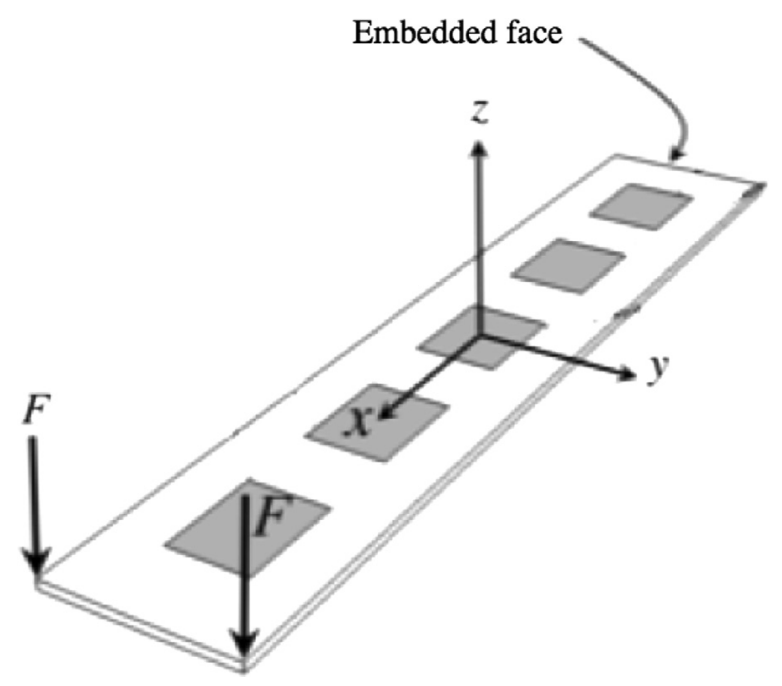

Fig. 18. Cantilever antenna's boundary conditions.

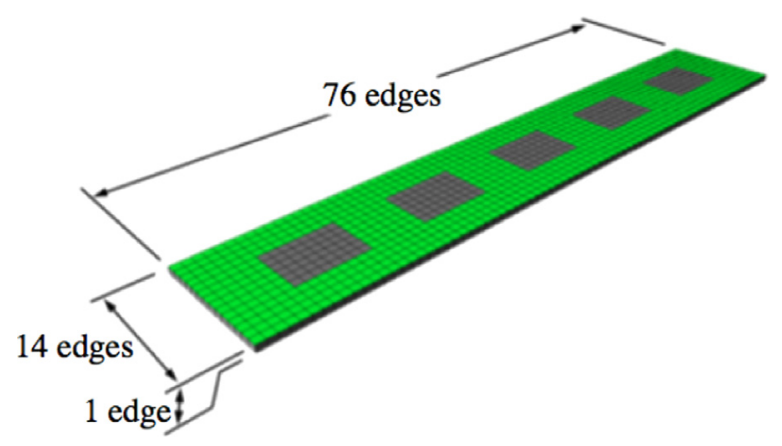

Fig. 19. Meshed antenna.

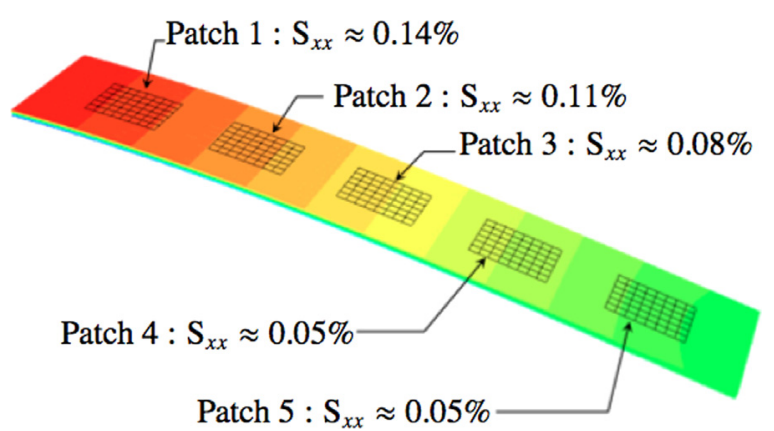

Fig. 20. $S_{x x}$ strains along the antenna.

The $S_{x x}$ strains, obtained using $76 \times 14 \times 1$ edges mesh (see Fig. 19), are presented in Fig. 20. As expected, the maximal strains are located near the embedding.

The influence of the mechanical strains on the input impedances and reflexion coefficients are respectively shown in Figs. 21 and 22 for three probes: probe 1 , close to the embedding, probe 2 , in the center of the antenna and probe 5 , close to the free edge. In these figures, dark curves refer to the planar configuration while orange ones refer to distorted antenna. In Fig. 21 solid and dotted curves respectively represent real and imaginary parts of the input impedances.

As previously observed, the patches extension leads to curve shifts toward the left (lower frequencies). On the other hand, these strains have no effect on the antenna bandwidth. Indeed, considering, for 

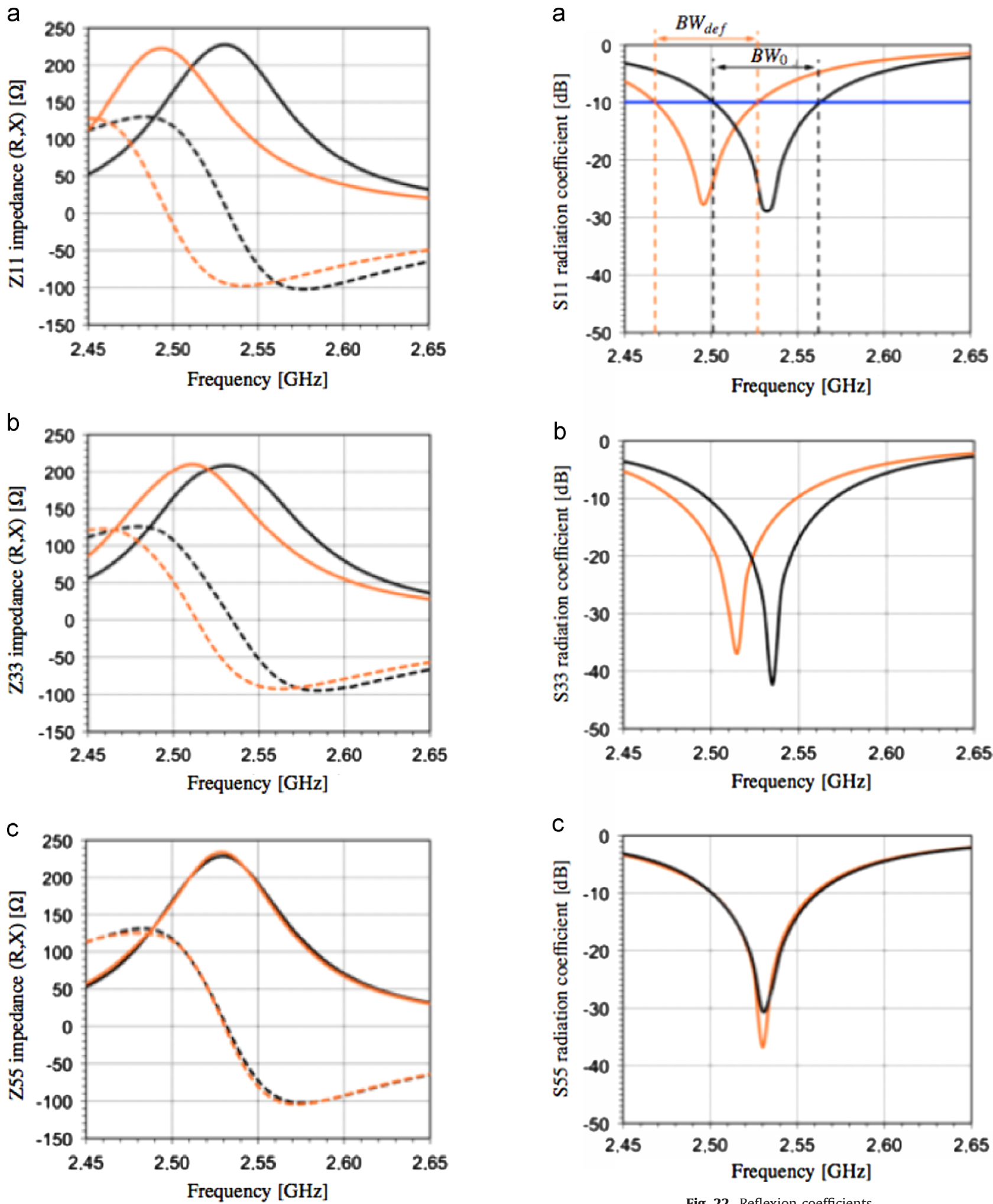

Fig. 21. Input impedances.

example, Fig. 22(a), the calculated planar and deformed bandwidth are equal to $B W_{0}=B W_{\text {def }}=2 \%$.

Moreover, the curve shifts are proportional to the patch strains state: shift is maximal for probe 1 corresponding to the more strained patch and curves are merged for probe 5 where strains are nearly null.

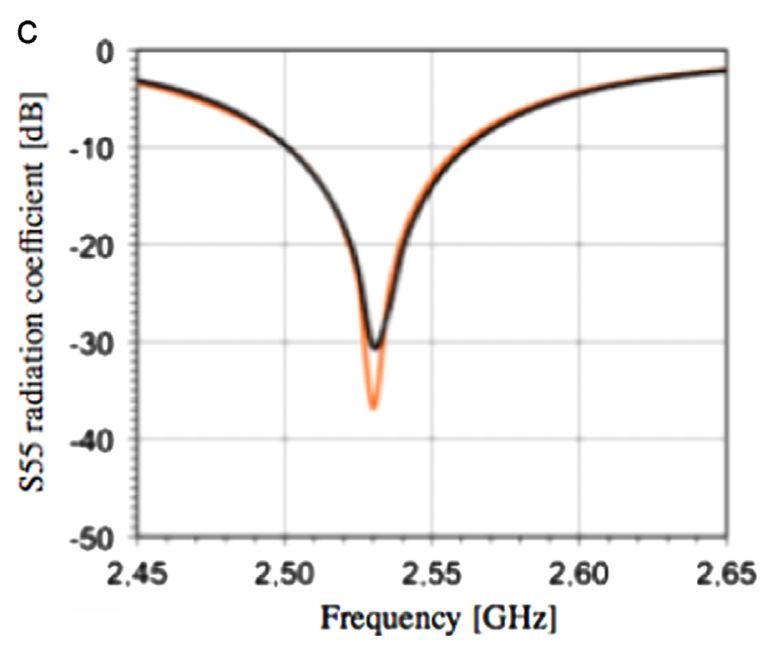

Fig. 22. Reflexion coefficients.

Finally, Figs. 23 and 24 respectively present the total gain of polar radiating diagrams obtained for a $2.5 \mathrm{GHz}$ excitation frequency, in the E-plane $((x, y)$ plane $)$ and the H-plane $((y, z)$ plane). As the main strains are in the $x$ direction, the fluctuation of the H-plane radiation pattern is not significant. On the other hand, the deformed shape of the structure induces a tilt about $10^{\circ}$ compared to the planar state. 


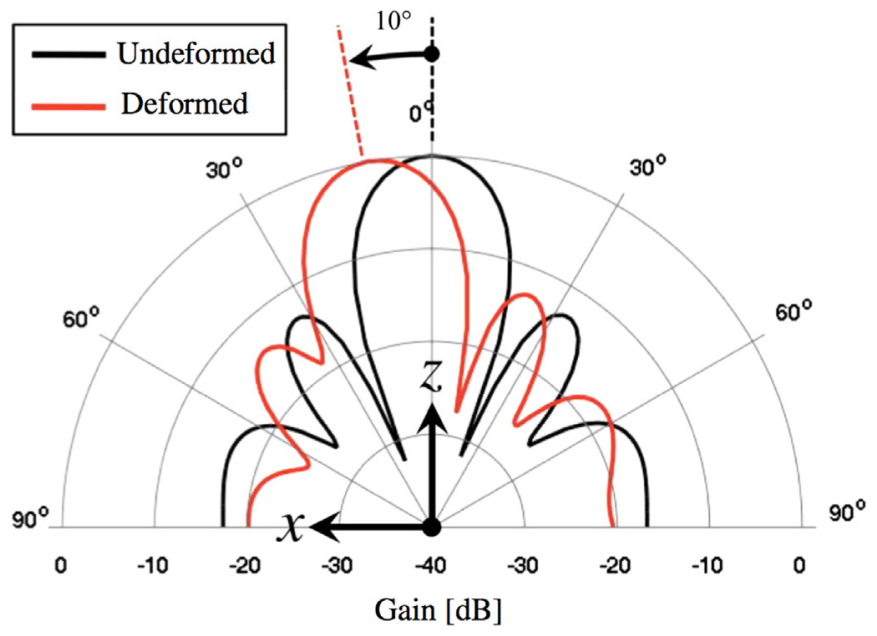

Fig. 23. E-plane radiation diagrams.

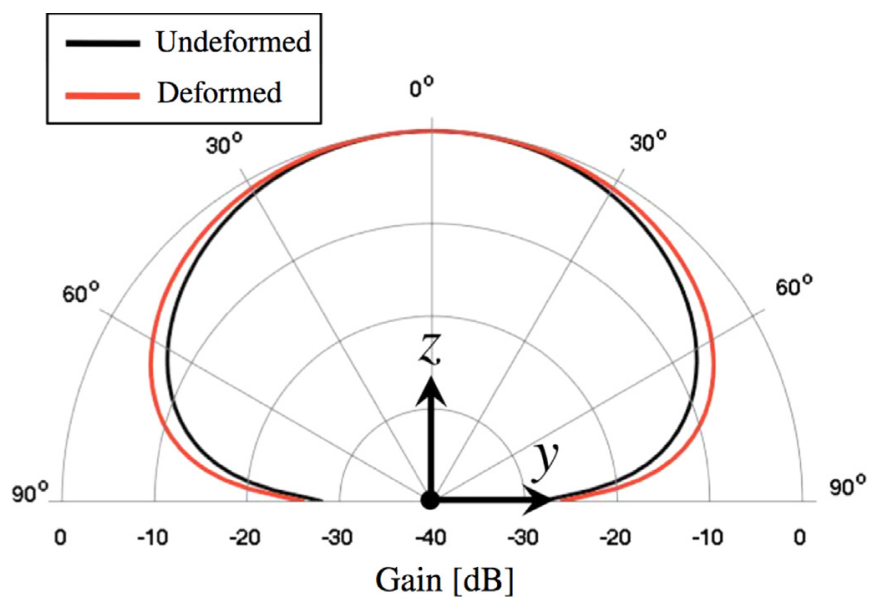

Fig. 24. H-plane radiation diagrams.

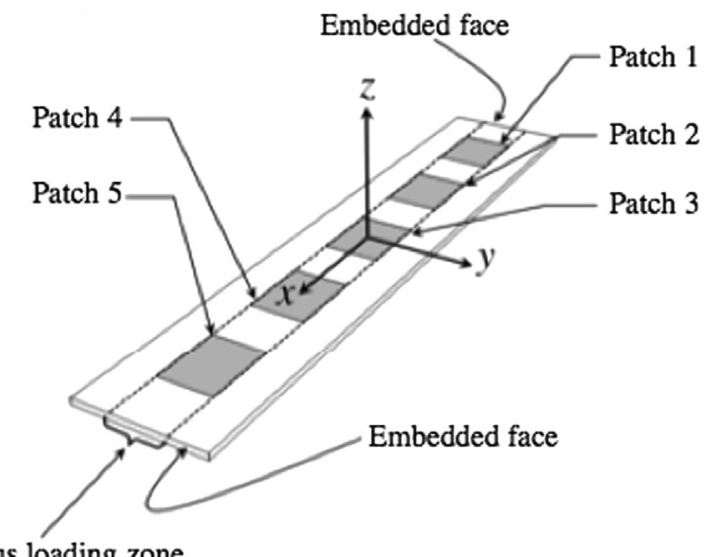

Sinus loading zone

Fig. 25. Clamped-clamped antenna's boundary conditions.

\subsubsection{Clamped-clamped antenna}

The previous antenna is now clamped at $x=-L / 2$ and $x=L / 2$ and subjected to a sinusoidal pressure on the area defined in Fig. 25, and given by

$P=P_{0}\left|\cos \left(\frac{\pi x}{L}\right)\right|$.

where $P_{0}=0.015 \mathrm{MPa}$.

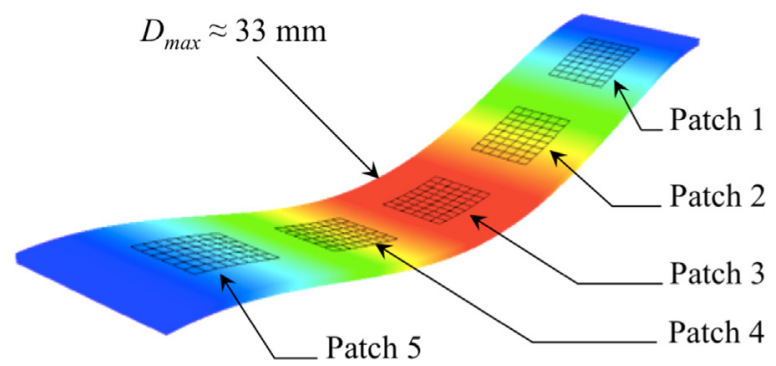

Fig. 26. Mechanical displacements on the deformed antenna.

The mechanical load and boundary conditions being symmetric in relation to the $(0, x, y)$ plane (Fig. 25), a similar symmetry is obtained for the deformed shape of the antenna, as presented in Fig. 26. Then, the maximal vertical displacement is obtained at the center line of the structure and is about $33 \mathrm{~mm}$.

Fig. 27 presents the input impedances obtained on probes 1, 3 and 5. Dark curves refer to the planar configuration while light ones refer to distorted antenna. Moreover, solid and doted curves respectively represent real and imaginary parts of the input impedances.

Patches 1 and 5 being symmetric in relation to the $(0, x, y)$ plane whatever the considered shape state, similar curves are obtained for probes 1 and 5. Obviously, similar observations can be noted for patches 2 and 4 .

Furthermore, it is to be pointed out that the impact of the antenna distortion on probe 3 (center of he structure) input impedances lies in an increase in amplitudes.

This point could be explained by observing Figs. 28 and 29 which respectively show the total gain of polar radiating diagrams obtained for a $2.5 \mathrm{GHz}$ excitation frequency, in the E-plane $((x, y)$ plane $)$ and the $\mathrm{H}$-plane $((y, z)$ plane). Indeed, given the symmetric deformed shape of the antenna no tilt is observed here. On the other hand, the antenna distortion impacts the minima on the E-plane radiation pattern around $55^{\circ}$. Actually, their increase leads to the merge of the secondary lobes and to an increase of the transmitted power. Moreover, similar to the previous test, the fluctuation of the H-plane radiation pattern is not significant.

Finally, it is to be underlined that similar results can be observed for reflexion coefficients (not presented in this paper).

\subsection{Four patches antenna conformed on a sphere}

Finally, an antenna made of four $20 \mathrm{~mm} \times 30 \mathrm{~mm}$ patches lying on the top of a $80 \mathrm{~mm} \times 120 \mathrm{~mm}$ spherical dielectric filled cavity is considered. Geometric characteristics are given in Fig. 30. The substrate dielectric permittivity and Young modulus respectively equal to 2.17 and $1 \mathrm{GPa}$.

The influence of spherical conformation will hereafter be analyzed for three sphere radius:

- $R=\infty$ (planar antenna),

- $R=10 \mathrm{~cm}$,

- $R=5 \mathrm{~cm}$.

A similar mesh, presented in Fig. 31 for the specific conformation radius equal to $R=5 \mathrm{~cm}$, is used for all calculations.

Whatever the considered configuration the shape of the antenna is characterized by two symmetry planes: $(0, x, z)$ and $(0, y, z)$. Therefore, identical input impedances and reflexion coefficients can be observed for the four probes. Results obtained for probe 1 are presented in Fig. 32 .

As previously observed ( 2 patches antenna test), the structure conformation leads to significants effects on the antenna behavior. Indeed, more conformed is the antenna (lower is the sphere 

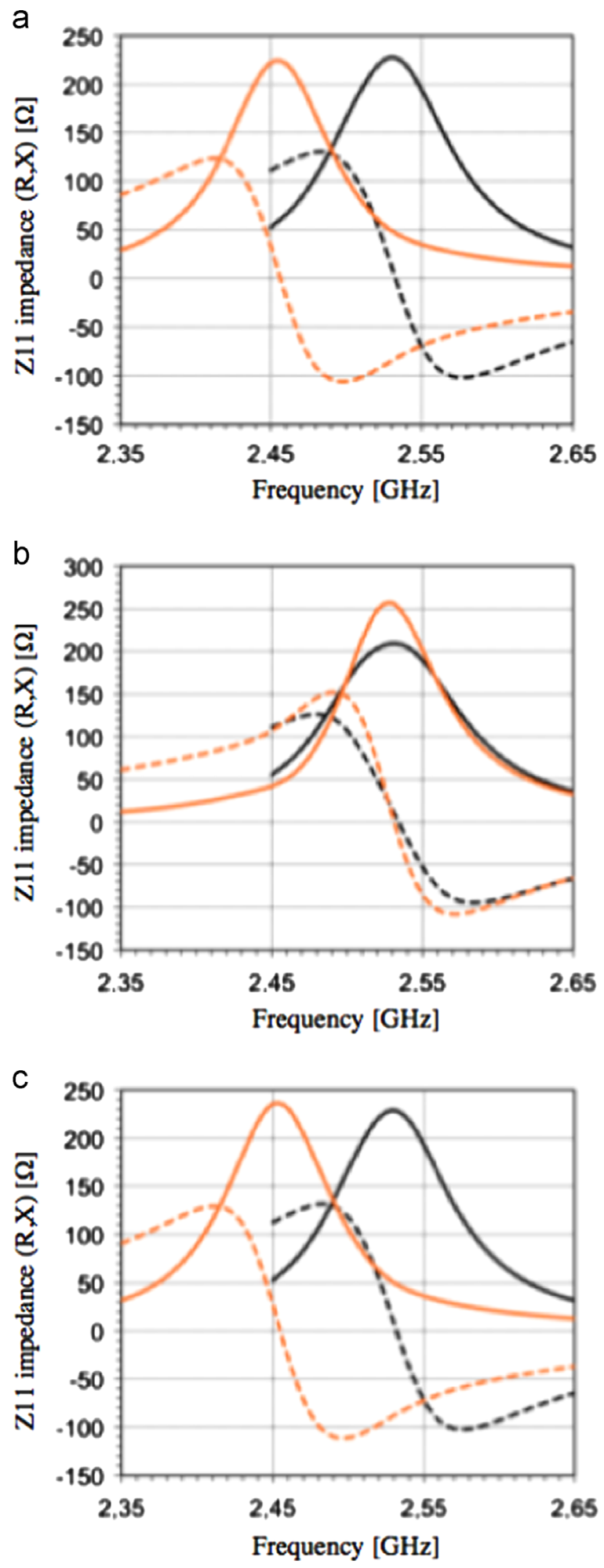

Fig. 27. Input impedances.

radius), greater is the shift in the curves and thus greater is the resonance frequency.

On the other hand, radiating diagrams of each of the three conformation radii have been computed for their resonance frequency (obtained from Fig. 32). The 3D graphics are proposed in Figs. 33-35. One can note that the two important secondary lobes observed in the planar case decrease while the conformation radius increases. This is clearly highlighted in the polar radiating diagrams plotted in Figs. 36 and 37.

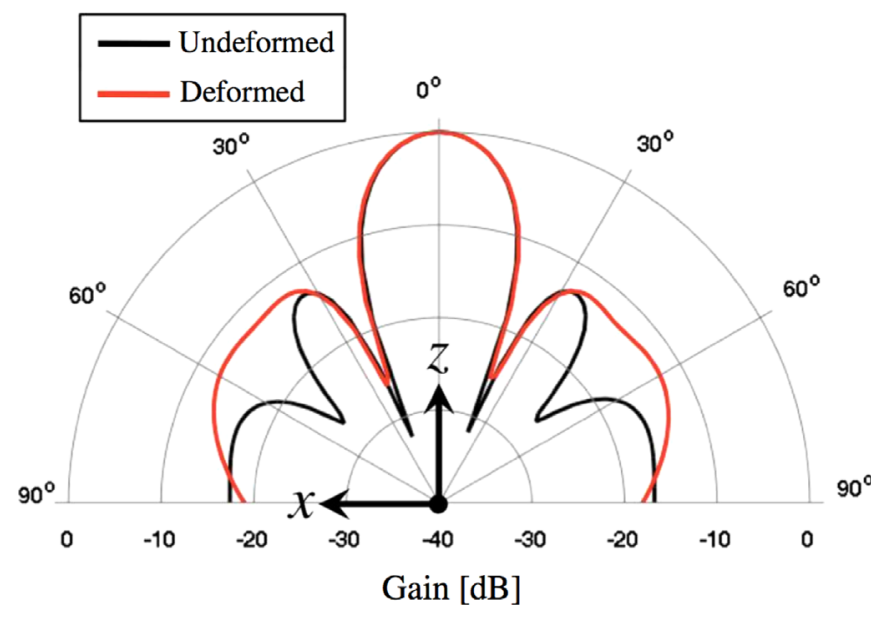

Fig. 28. Radiation pattern in the E-plane.

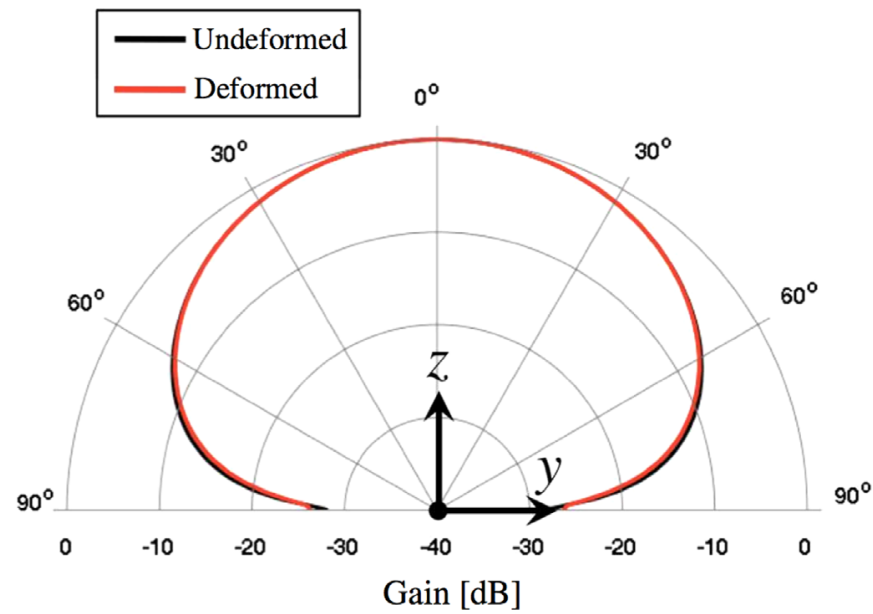

Fig. 29. Radiation pattern in the H-plane.

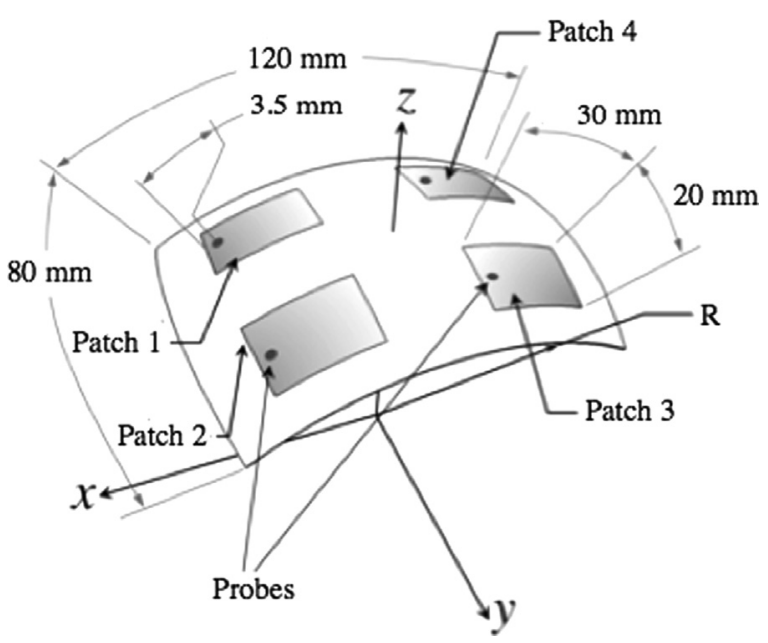

Fig. 30. 4 patches antenna spherically conformed.

\section{Conclusion}

This work deals with the modeling of the electromagnetic radiative patch antennas subjected to mechanical stresses. It has been motivated by the MSIE project from the competitiveness French Cluster Astech, where the development of metamaterial strip antennas for aeronautical application is evaluated. Such antennas which are thin and conformable can be embedded on 


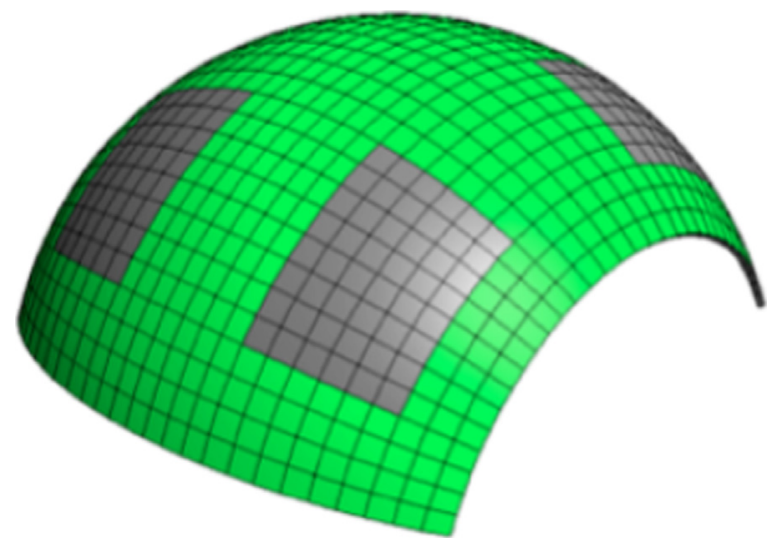

Fig. 31. Meshed antenna $(R=5 \mathrm{~cm})$.
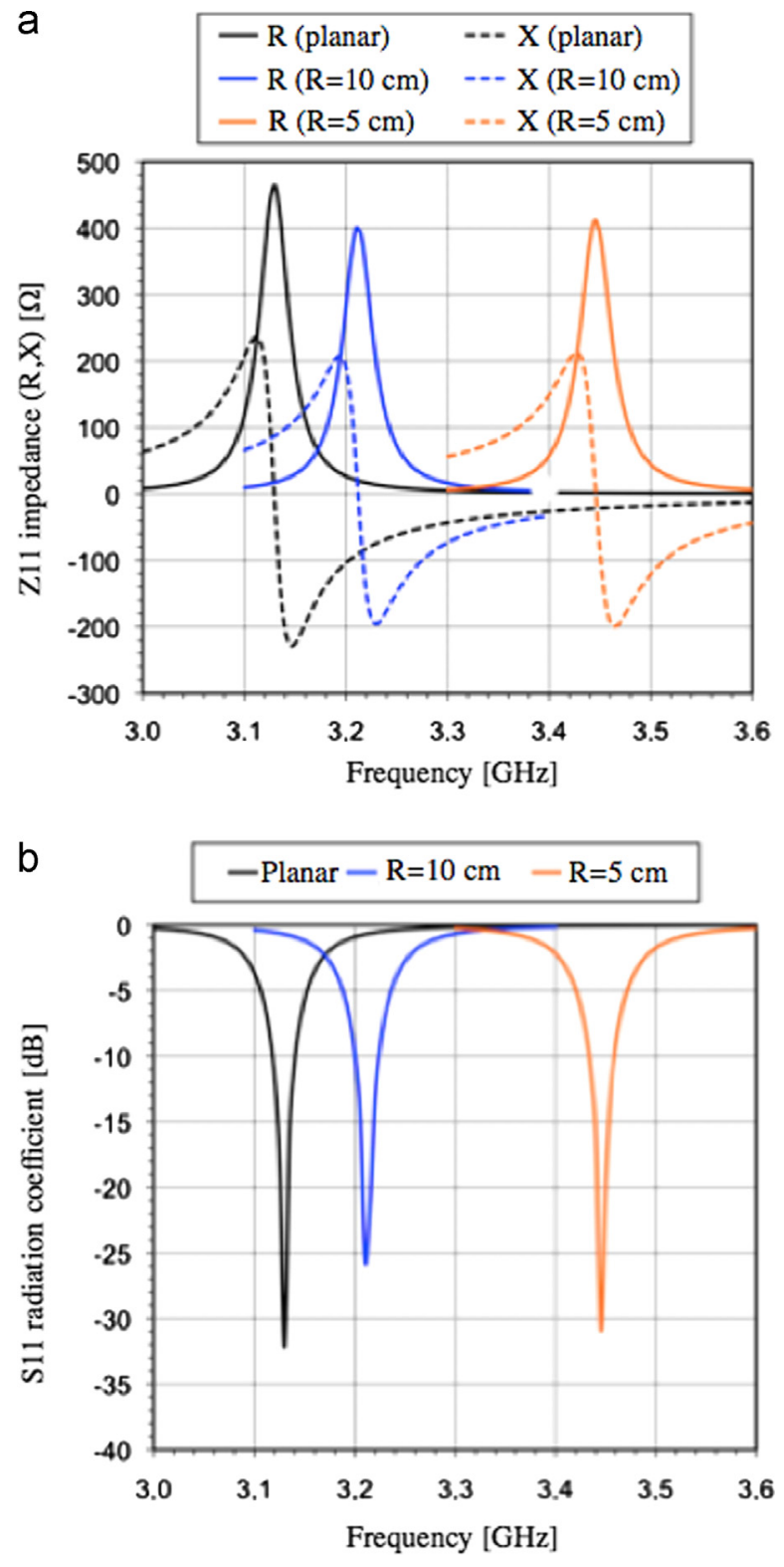

Fig. 32. Input impedances and reflexion coefficient for probe 1.

any aircrafts surfaces, subjected to mechanical loads. Consequently, the influence of mechanical distortions on electromagnetic signals has to be evaluated.
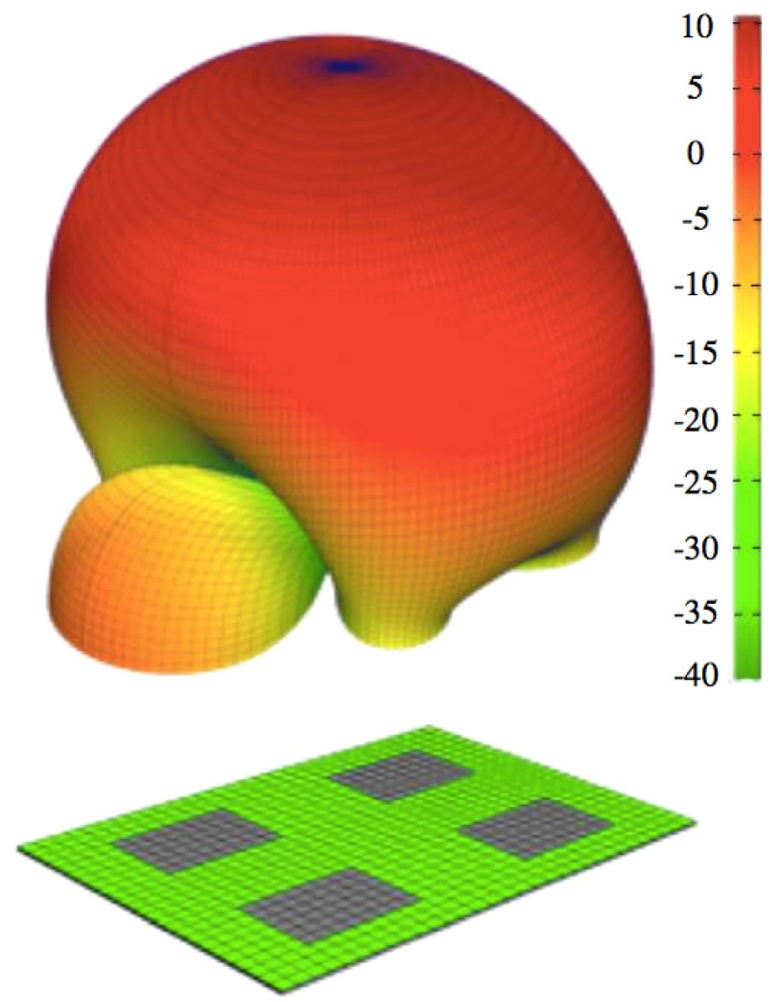

Fig. 33. The radiation patterns for the planar configuration.

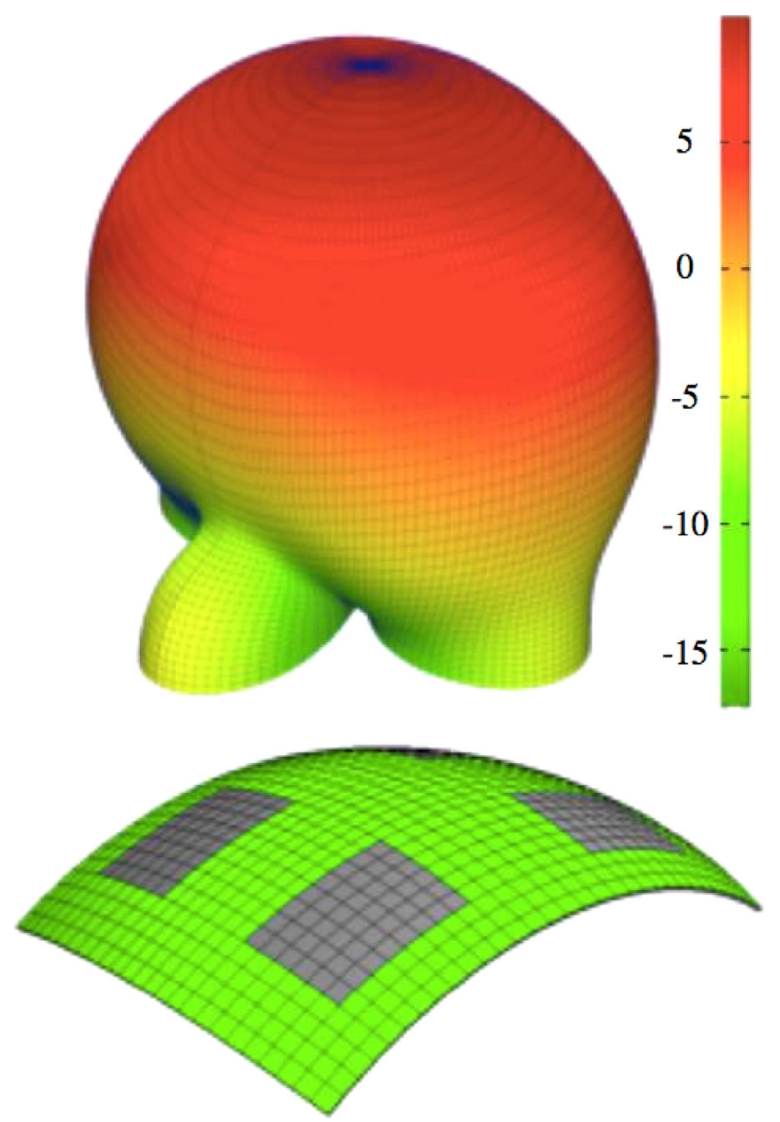

Fig. 34. The radiation patterns for $R=10 \mathrm{~cm}$.

In this way, a recent numerical tool is presented in this paper, taking into account the weak coupling between electromagnetism and mechanical behavior to model radiative microstrip antenna. 


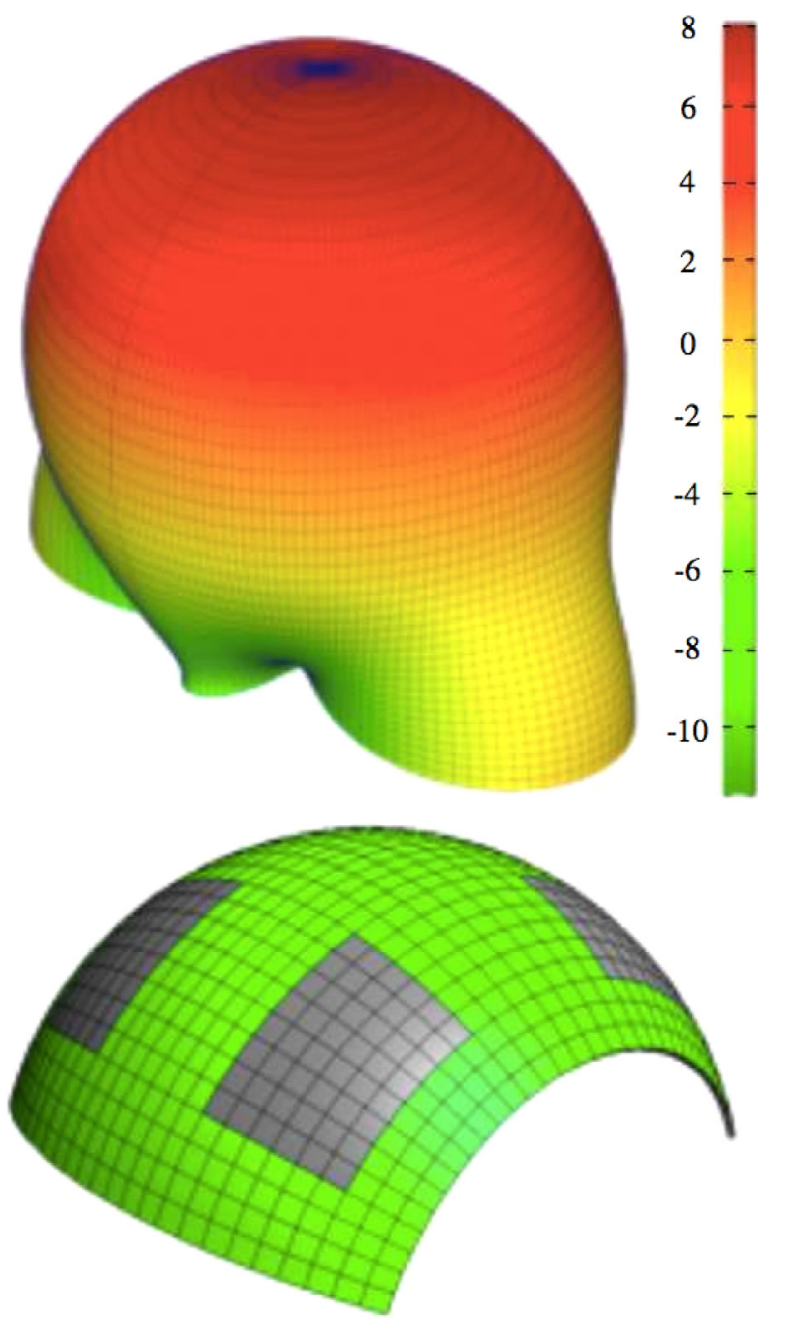

Fig. 35. The radiation patterns for $R=15 \mathrm{~cm}$.

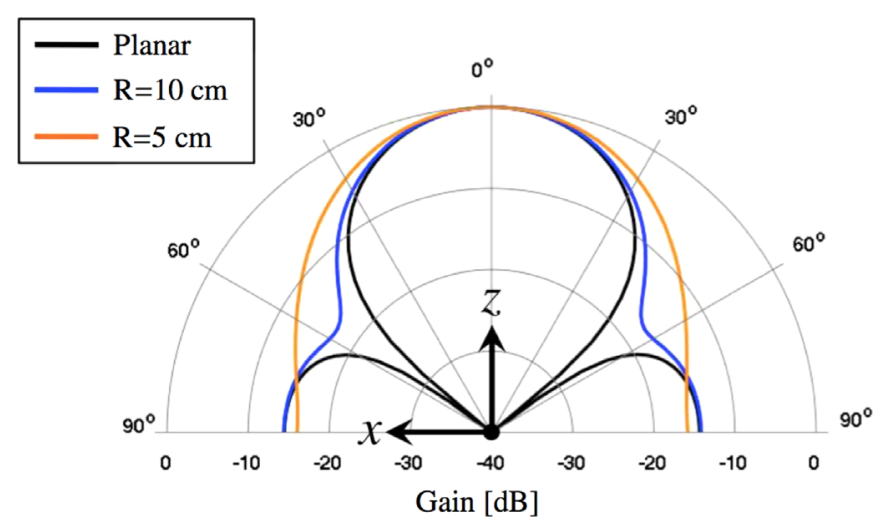

Fig. 36. The radiation pattern in the E-plane.

An hexahedral finite element is developed with a classical nodal formulation for mechanical fields while the electromagnetic ones are expressed by an edge formulation with vector finite elements. From the weak formulation, a FEM/BIM approach is used to avoid the discretization of overall space. It consists of introducing a fictitious boundary that encloses the structure to be studied. Classical FEM is used to approximate the fields in the closed domain, whereas the fields in the opened region are evaluated by the BIM.

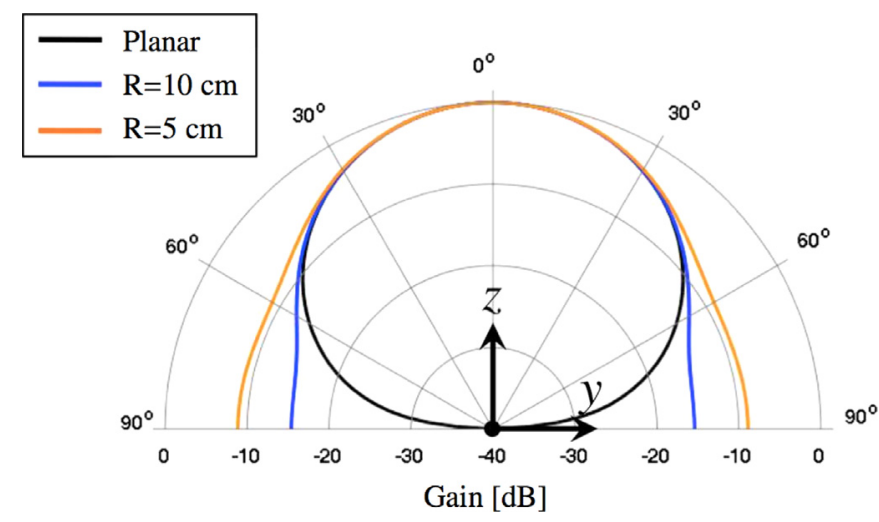

Fig. 37. The radiation pattern in the H-plane.

The numerical simulations presented in this paper show that the effect of mechanical strains on the electromagnetic signals is significant in these studies. Experimental measurements on a warped antenna subjected to bending and twisting loads are in progress. They confirm results presented here [1]. From this observation, techniques of active control of the electromagnetic signals should be added to correct the electromagnetic behavior of the strained antennas.

\section{Appendix A. The basis functions for the hexahedral edge element}

The expression of the shape functions along each edge of the hexahedral element are [6]

$\overrightarrow{W_{k}^{v}}(\xi, \eta, \zeta)=\frac{l_{k}^{e}}{8}\left(1+\eta_{k} \eta\right)\left(1+\zeta_{k} \zeta\right) \overrightarrow{\text { grad }} \xi$ for edges parallel to $\vec{\xi}$

$\overrightarrow{W_{k}^{v}}(\xi, \eta, \zeta)=\frac{l_{k}^{e}}{8}\left(1+\xi_{k} \xi\right)\left(1+\zeta_{k} \zeta\right) \overrightarrow{\operatorname{grad}} \eta$ for edges parallel to $\vec{\eta}$

$\overrightarrow{W_{k}^{v}}(\xi, \eta, \zeta)=\frac{l_{k}^{e}}{8}\left(1+\eta_{k} \eta\right)\left(1+\xi_{k} \xi\right) \overrightarrow{\operatorname{grad}} \zeta$ for edges parallel to $\vec{\zeta}$,

where $\xi_{k}, \eta_{k}, \zeta_{k}$ denotes the edge's location in $(0, \hat{\xi}, \hat{\eta}, \hat{\zeta})$ and $l_{k}{ }^{e}$ denotes its length.

\section{References}

[1] Han X, Adnet N, Bruant I, Pablo F, Ouslimani H, Proslier L, et al. Experimental study of the behavior of an EBG-based patch antenna subjected to mechanical deformations. Progr Electromagn Res B 2013;48:313-27.

[2] You CS, Hwang W. Design of load-bearing antenna structures by embedding technology of microstrip antenna in composite sandwich structure. Compos Struct 2005;71:378-82.

[3] Antilla GE, Alexopoulos NG. Scattering from complex three dimensional geometries by a curvilinear hybrid finite element integral equation approach. J Opt Soc Am A 1994;11(4):1455-7.

[4] Jacobs AKRT. Analysis of planar and curved microstrip antennas. J Microwaves Optoelectron 2007;6(1):96-110.

[5] Khedrouche D, Bouttout F, Fortaki T, Benghalia A. Spectral-domain analysis of multilayer cylindrical-rectangular microstrip antennas. Eng Anal Boundary Elem 2009;33:930-9.

[6] Volakis JL, Chatterjee A, Kempel LC. Finite element method for electromagnetics. Antennas, microwave circuits and scattering applications. IEEE Press; 1998.

[7] Reig B, Chevrier J, Lambrecht A, Greffet J, Leconte V. Monaco: Modélisation nano composants. In: Poster presentation in J3N2009; 2009.

[8] Su C, Ke H, Hubbing T. 25th annual review of progress in applied computational electromagnetics. Overview of electromagnetic modeling software; 2009.

[9] Adnet N. Modélisation numérique du couplage mécanique/électromagnétisme pour l'étude de la sensibilité du comportement électromagnétique d'antennes patch aux déformations mécaniques [Ph.D. thesis]. Université Paris Ouest Nanterre La Défense; 2012.

[10] Bossavits A. Computational electromagnetism, variational formulation, complementary, edge elements. Academic Press; 1998. 
[11] Jin J. The finite element method in electromagnetics. 2nd edition. WileyInterscience; 2002.

[12] Fung YC. Foundations of solid mechanics. Prentice-Hall, Inc.; 1965.

[13] Release 11.0 documentation for Ansys; 2011.

[14] Silvester PP, Ferrari RL. Finite element for electrical engineers. Cambridge University Press; 1990.

[15] Demkowicz L, Vardapetyan L. Modeling of electromagnetic absorption/scattering problems using hp-adaptive finite elements. Comput Methods Appl Mech Eng 1998; 152:103-24.

[16] Tai CT. Dyadic Green's function in electromagnetic theory. 2nd editionIEEE Press; 1993 p. 1.

[17] Eibert TF, Volakis JL, Wilton DR, Jackson DR. Hybrid Fe/Bi modeling of 3-D doubly periodic structures utilizing triangular prismatic elements and an MPIE formulation accelerated by the Ewald transformation. IEE Trans Antennas Propag 1999;47(5):843-50.

[18] Volakis JL, Sertel K, Jorgensen E, Kindt RW. Hybrid finite element and volume integral methods for scattering using parametric geometry. Comput Modeling Eng Sci 2000;1(1):11-24.

[19] Conil E. Propagation électromagnétique en milieu complexe [Ph.D. thesis]. Institut National Polytechnique de Grenoble; 2005.

[20] Krantz RG, Parks HR. Geometric integration theory. Birkhauzer Verlag; 2008.

[21] Andersen LS, Volakis JL. Adaptive multiresolution antenna modeling using hierarchical mixed-order tangential vector finite elements. IEE Trans Antennas Propag 2001;49(2):211-22.
[22] Nédélec J-C. A new family of mixed elements in $r^{3}$. Numer Math 1986;50:57-81.

23] Eibert TF, Sertel K, Volakis JL. Hybrid finite element modelling of conformal antenna and array structures utilizing fast integral methods. Int J Numer Modelling: Electron Netw Devices Fields 2000;13:81-101.

[24] Volakis JL, Andersen LS, Eibert TF, Sertel K, Li Z. Fast hybrid finite element methods and their applications for conformal antennas. Ann Arbor, USA: Radiation Laboratory, EECS Department, University of Michigan; 1999.

[25] Forrai DP, Newma EH. Radiation and scattering from loaded microstrip antennas over a wied bandwidth. Technical Report 719493-1. Ohio State University; 1988

[26] Adnet N, Bruant I, Pablo F, Proslier L. Influence of mechanical strains on electromagnetic signals of microstrip antenna. In: FEM/BIM model, 4th international conference on computational methods for coupled problems in science and engineering, Kos-Island Greece; 2011.

[27] Balanis CA. Antenna theory: analysis and design. 3rd edition. WileyInterscience; 2005.

[28] Volakis JL. Antenna engineering handbook. 4th editionMcGraw-Hill Professional; 2007.

[29] Elrashidi A, Elleithy K, Bajwa H. The fringing and resonance frequency of cylindrical microstrip printed antenna as a function of curvature. Int J Wireless Commun Networking 2011;3(2):155-62. 